Research in Astron. Astrophys. Vol.0 (200x) No.0, 000-000

http://www.raa-journal.org http://www.iop.org/journals/raa

Key words: stars: variables : other - galaxies: individual (LMC)

\title{
Analysis of a selected sample of RR Lyrae stars in LMC from OGLE III
}

\author{
B.-Q. Chen ${ }^{1,2}$, B.-W. Jiang ${ }^{1}$ and M. Yang ${ }^{1}$ \\ 1 Department of Astronomy, Beijing Normal University, Beijing 100875, \\ P.R.China;bchen@mail.bnu.edu.cn,bjiang@bnu.edu.cn,myang@mail.bnu.edu.cn \\ 2 Institut Utinam, CNRS UMR6213, OSU THETA, Université de Franche-Comté, 41 bis avenue de \\ l'Observatoire, 25000 Besançon, France
}

\begin{abstract}
A systematic study of RR Lyrae stars is performed based on a selected sample of 655 objects in the Large Magellanic Cloud with observation of long span and numerous measurements by the Optical Gravitational Lensing Experiment III project. The Phase Dispersion Method and linear superposition of the harmonic oscillations are used to derive the pulsation frequency and variation properties. It is found that there exists an Oo I and Oo II dichotomy in the LMC RR Lyrae stars. Due to our strict criteria to identify a frequency, a lower limit of the incidence rate of Blazhko modulation in LMC is estimated in various subclasses of RR Lyrae stars. For fundamental-mode RR Lyrae stars, the rate $7.5 \%$ is smaller than previous result. In the case of the first-overtone RR Lyr variables, the rate $9.1 \%$ is relatively high. In addition to the Blazhko variables, fifteen objects are identified to pulsate in the fundamental/first-overtone double mode. Furthermore, four objects show a period ratio around 0.6 which makes them very likely the rare pulsators in the fundamental/second-overtone double-mode.
\end{abstract}

\section{INTRODUCTION}

RR Lyrae stars (RRLS) are pulsating variables on the horizontal branch in the H-R diagram. They have short periods of 0.2 to 1 day and low metal abundances $Z$ of 0.00001 to 0.01 . Usually they can be easily identified by their light curves and color-color diagrams (Li et al., 2011). RRLS is famous for the "Blazhko effect" (Blažko, 1907), a periodic modulation of the amplitude and phase in the light curves, which is still a mystery in theory today. The main photometric feature of the Blazhko effect is that the frequency spectra of the light curves are usually strongly dominated by a symmetric pattern around the main pulsation frequency $f_{0}$, i.e., $k f_{0}$ and $k f_{0} \pm f_{\mathrm{BL}}$ where $f_{\mathrm{BL}}$ is the modulation frequency and $k$ is the harmonic number (Kovács, 2009). Moreover, the higher-order multiplets such as quintuplets and higher, i.e. multiplets $k f_{0} \pm l f_{\mathrm{BL}}$, are now also attributed to the Blazhko effect (Benkő et al., 2011). On the other hand, the amplitudes of modulation components are usually asymmetric so that one side could be under the detection limit in highly asymmetric cases, which may lead to the asymmetric appearance of the frequency spectra. Several models are proposed to explain this effect, including the non-radial resonant rotator/pulsator (Goupil \& Buchler, 1994), the magnetic oblique rotator/pulsator (Shibahashi, 2000), 2:1 resonance model (Borkowski, 1980), resonance between radial and non-radial mode (Dziembowski \& Mizerski, 2004), 9:2 resonance model (Buchler \& Kolláth, 2011) and convective cycles model (Stothers, 2006). However, none of them is able to interpret all the observational phenomena about the Blazhko effect. 
A systematic study of RRLS helps to understand their nature, such as the incidence rate of various pulsation modes, the distribution of modulation frequency and amplitude and the dependence of the variation properties on environment. This has been performed on the basis of some datasets with large amount of data for variables. The early micro-lensing projects, MACHO (Alcock et al., 2003) and OGLE (Soszynski et al., 2008) that surveyed mainly LMC, SMC and the Galactic bulge, and the variables-oriented all-sky survey project, ASAS (Szczygieł \& Fabrycky, 2007), have given particularly great support to such study. Using the MACHO data, Alcock et al. (2000) and Nagy \& Kovács (2006) analyzed the frequency of 1300 first overtone RRLS in LMC with an incidence rate of the Blazhko variables of 7.5\%. Meanwhile, Alcock et al. (2003) made a frequency analysis of 6391 fundamental mode RRLS in LMC that resulted in an incidence rate of the Blazhko variables of $11.9 \%$. With the OGLE-I data, Moskalik \& Poretti (2003) searched for multiperiodic pulsators among 38 RRLS in the Galactic Bulge. Mizerski (2003) made a complete search for multi-period RRLS from the OGLE-II database, while Collinge et al. (2006) presented a catalogue of 1888 fundamental mode RRLS in the Galactic bulge from the same database.

Recently, Moskalik \& Olech (2008) conducted a systematic search for multiperiod RRLS in $\omega$ Centauri, a globular cluster, and found the incidence rate of Blazhko modulation pretty high, about $24 \%$ and $38 \%$ for the fundamental and first-overtone RRLS respectively. Jurcsik et al. (2009) got a 47\% incidence rate in the dedicated Konkoly survey sample of 30 fundamental-mode RRLS in the Galactic field. Kolenberg et al. (2010) also claimed at least 40\% RRLS in the Kepler space mission sample of 28 objects exhibiting the modulation phenomenon.

The OGLE-III database released in 2009 (Soszyński et al., 2009) contained 24906 light curves that are preliminarily classified as RRLS in the Large Magellanic Cloud, the ever largest sample of RRLS. This database covers a time span of about 10 years that makes a large-scale analysis of the RRLS variation possible. Soszyński et al. (2009) analyzed the basic statistical features of RRLS in the LMC and divided them into 4 subtypes: RRab, RRc, RRd and RRe. With this sample of RRLS, the study of the structure of LMC was developed. Peicha \& Stanek (2009) investigated the structure of the LMC stellar halo; Subramaniam \& Subramanian (2009) found that RRLS in the inner LMC trace the disk and probably the inner halo; Feast et al. (2010) established a small but significant radial gradient in the mean periods of Large Magellanic Cloud (LMC) RR Lyrae variables. The data of RRLS in SMC and the Galactic bulge are also released (Soszyñski et al., 2010; Soszyński et al., 2011) and some work is also done with those data (Pietrukowicz et al., 2011). It is worth to note that these work mainly deals with the structure of LMC other than the RR Lyr variables.

In this paper, we focus the study on the RR Lyr variables themselves based on the released OGLE-III database. With the PDM and Fourier fitting methods, we make a precise systematic frequency analysis of carefully selected 655 RRLS in LMC, and a detailed classification of the RRLS in the LMC based on which to discuss the incidence rate of the Blazhko modulation in various pulsation modes. The data and the sample are illustrated in section 2, the method is introduced in section 3, the detailed classification of RRLS in LMC in section 4 and discussion in section 5.

\section{THE SAMPLE}

Soszyński et al. (2009) presented a catalog of 24,906 RR Lyrae stars discovered in LMC based on the OGLE-III observations and classified them into 17,693 fundamental-mode, 4958 first-overtone, 986 double-mode and 1269 suspected second-overtone RRLS. Thanks to their generosity, all the data are released. This catalog has three columns recording the observational Julian Date, magnitude in the I or $\mathrm{V}$ band and error of the magnitude. Since the number of measurements in the $\mathrm{V}$ band is much fewer than in the I band, our analysis mainly makes use of the I-band data. For over 20,000 objects, precise analysis of the frequency for all of them seems an improbable task. Fortunately, the statistical properties can be reflected by a much smaller sample. Thus, we concentrate our study on a sample of RRLS that were measured as many times as possible with high precision.

As the amplitude of some RRLS is rather small as about $0.1 \mathrm{mag}$, the measurements with assigned photometric error bigger than $0.1 \mathrm{mag}$ are dropped. In the released database, the number of measure- 
Table 1 List of the RRLS in our sample. The full table is available in electronic form at the CDS.

\begin{tabular}{|c|c|c|c|c|c|c|c|c|c|}
\hline ID & OGLE name & RA & Dec & $N_{\text {ori }}$ & $N_{\text {cor. }}$. & $I$ & $V$ & subtype & MACHO ID \\
\hline 1 & OGLE-LMC-RRLYR-00762 & $04: 45: 14.61$ & $-68: 21: 28.2$ & 1023 & 1016 & 18.831 & 19.560 & RR0 & \\
\hline 2 & OGLE-LMC-RRLYR-05348 & 05:07:31.21 & $-69: 11: 05.3$ & 1179 & 1176 & 18.549 & 18.948 & RR1 & 1.4412 .1128 \\
\hline 3 & OGLE-LMC-RRLYR-05901 & 05:09:04.43 & $-69: 36: 07.0$ & 1030 & 1009 & 18.825 & 19.341 & RR01 & \\
\hline 4 & OGLE-LMC-RRLYR-05976 & $05: 09: 15.63$ & $-69: 18: 48.1$ & 1035 & 1033 & 18.701 & 19.347 & RR0 & 1.4652 .1639 \\
\hline 5 & OGLE-LMC-RRLYR-05990 & 05:09:17.09 & $-69: 21: 13.8$ & 1045 & 1031 & 18.876 & 19.406 & RR0 & 5.4652 .8925 \\
\hline 6 & OGLE-LMC-RRLYR-06004 & 05:09:19.04 & $-69: 28: 47.5$ & 1035 & 1028 & 18.723 & 19.240 & RR0 & \\
\hline 7 & OGLE-LMC-RRLYR-06033 & 05:09:23.61 & $-69: 31: 17.8$ & 1010 & 1010 & 18.056 & 18.691 & RR0 & \\
\hline 8 & OGLE-LMC-RRLYR-0 & 05:09:34.62 & $-68: 4$ & 1029 & 1027 & 18.788 & 19.448 & RR0 & 32.3916 \\
\hline 9 & OGLE-LMC-RRLYR-06125 & 05:09:36.66 & $-68: 55: 30.5$ & 1032 & 1030 & 18.457 & 18.846 & RR0 & 79.4779 .1340 \\
\hline 10 & OGLE-LMC-RRLYR-06147 & 05:09:39.41 & $-68: 55: 07.4$ & 1017 & 1009 & 18.793 & 19.380 & RR0 & 79.4779 .2347 \\
\hline
\end{tabular}

ments is usually not as numerous as claimed in the OGLE-III catalog web. Most of them have fewer than 400 measurements, as can be seen in Fig. 1 that displays the distribution of the number of measurements for all the RRLS. In our sample, only the objects with more than 1000 measurements are kept. To exclude the foreground stars, the criterion $\bar{I} \geq 18 \mathrm{mag}$ is added. In the I/V-I diagram (Fig. 11), it can be seen that this brightness cutoff constrains the sources in the major RRLS area and excludes some sparse sources seemingly to be giant or foreground stars. With the limitation of brightness and number of measurements, our sample consists of 655 RRLS.

Through the color-magnitude diagram of the 655 RRLS in comparison with the complete group of all the 24906 RRLS sources identified by Soszyński et al. (2009) in Fig. 11(top panel), we can see that the sample agrees with the majority of RRLS. The miss of the faint sources (I > 19.5) is mainly due to our request of high photometry quality. In the same time, some red RRLS with V-I bigger than about 0.8 are neither included. The faint and red RRLS may be caused by extinction as they coincide pretty well with the $A_{V}=1$ trend (the extinction law is taken from Mathis (1990)) in Fig. 1. Thus the missed faint and red stars should have intrinsically similar brightness and color with the majority, and their absence in our sample shall not influence the statistical variation properties of RRLS. This sample of 655 RRLS has three advantages for the frequency analysis. Firstly, more than six-hundred stars are already big enough to obtain the statistical parameters objectively and to understand the common properties of RRLS in LMC. Secondly, the sample is not too big, so that we can not only make the frequency analysis accurately, but also check carefully for individual object, to make sure every result is reliable. Thirdly, the sample we compose contains the highest-quality data for RRLS in the OGLE-III project, and the derived variability properties should be highly reliable. As mentioned earlier, all stars in our sample have more than 1000 measurements. The time span is about 4000 days (i.e. close to 11 years), and the interval of two adjacent measurements is mostly shorter than 20 days. Theoretically, based on the effect of random and uncorrected noise, using the least square fit of a sinusoidal signal, we can roughly estimate the error of the frequency $10^{-7}$, and the error of amplitude $10^{-3}$ Montgomery \& Odonoghue, 1999). The objects in our sample are listed in Table 1, with the OGLE name, position, number of measurements both in original OGLE catalog and in our calculation, the magnitude in the I and V bands that are the average over all the measurements , the subtype of variation which will be discussed later, and MACHO ID if available. It should be noticed that the I magnitudes in the tables afterwards are the average value of the Fourier fitting.

\section{FREQUENCY ANALYSIS}

Most studies of the RRLS frequencies (e.g. Kolenberg et al. 2010) make use of the Period04 software that analyze the Fourier spectrum of the observed light curve. The advantage of Period04 is its high precision and intuitive power spectrum with both the frequency and amplitude shown, appropriate for analyzing multi-period light-curves. But, the Fourier analysis fits a sinusoidal signal, while for RRLS in the fundamental mode, their light-curves are very asymmetric. Besides, RRLS often have several harmonics, so that more than one period would be found for a single period RRLS. An alternative 

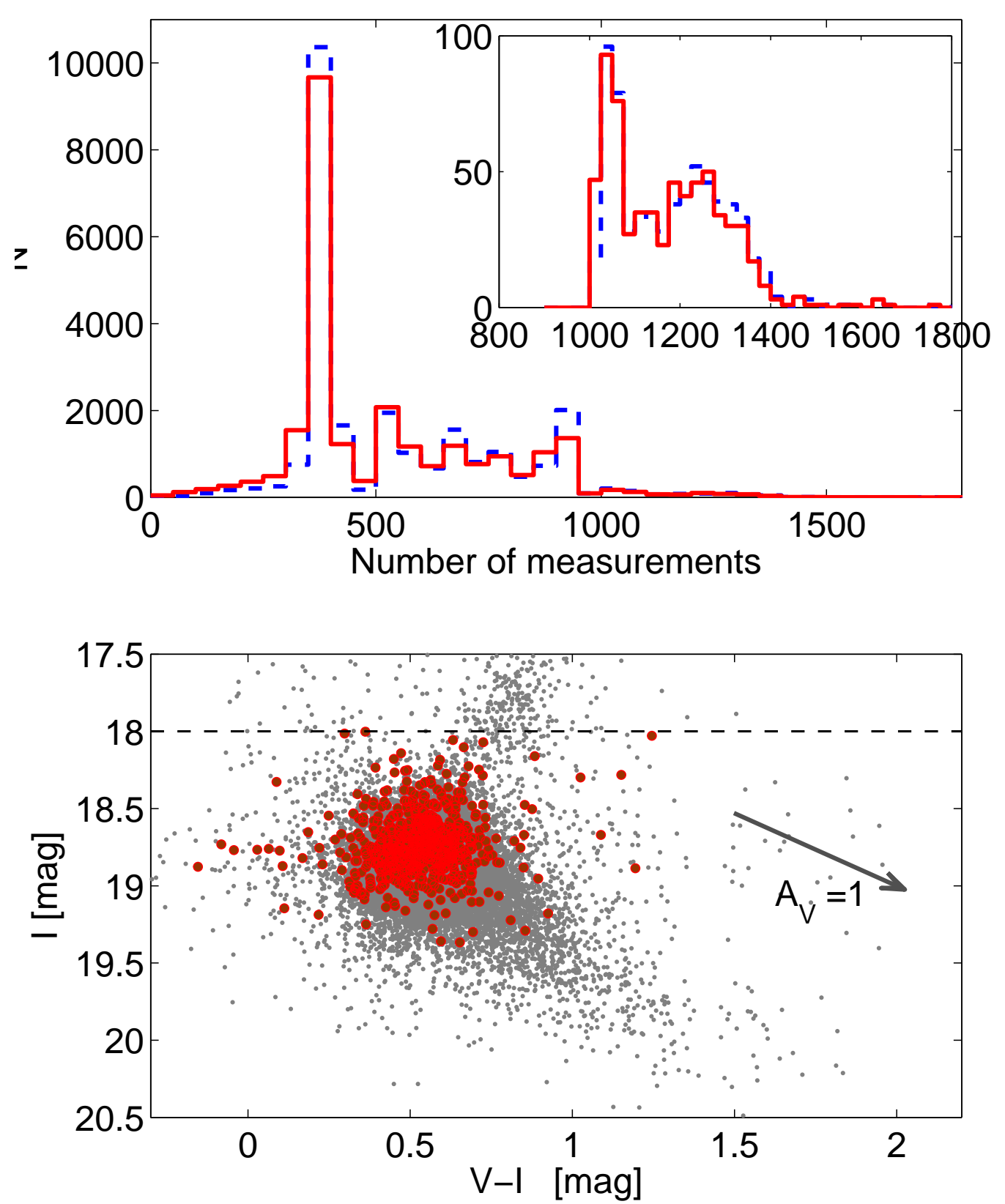

Fig. 1 Histogram of the number of measurements in the I band and the color-magnitude diagram of the OGLE III RRLS. Upper panel: the blue dash line is for the data originally from the catalog and the red solid line for those with photometric uncertainty smaller than 0.1 mag which is the threshold when selecting our sample; the inset is the histogram of our sample in which the photometric uncertainty is less than 0.1 mag and the number of measurements is more than 1000. Lower panel: the gray dots and red dots correspond to all the RRLS from OGLE III and those in our sample respectively; the dashed horizontal line at $\bar{I}=18 \mathrm{mag}$ is our criterion for brightness. The arrow stands for the $A_{V}=1$ vector. 
method to determine the frequency of light variation is the Phase Dispersion Method (PDM, Stellingwerf 1978) independent on the shape of the light curve or irregular distribution of measurements in the time domain. Although PDM also brings about a strong signal of the harmonics, this can be looked over in the folded phase curve, which needs a careful eye-check. The mediate volume of our sample makes it possible to check the phase curve one by one. Moreover, the result from PDM provides a mutual check between the two main-stream methods in the study of variable stars.

The PDM method looks for the right period of light variation in a range of trial periods by fixing the period of the minimum phase dispersion for the folded phase curve. The phase dispersion $\Theta_{\mathrm{PDM}}$ is defined as the ratio between the summed phase dispersion in all the phase bins to the phase dispersion of all the measurements. A perfect periodical light curve would produce $\Theta_{\mathrm{PDM}}=0$. The period of light variation of the RRLS sample is searched in two steps. In the first step, the frequency range is set to the whole range for RRLS variation, i.e. from $1 \mathrm{c} / \mathrm{d}$ to $5 \mathrm{c} / \mathrm{d}$, with a step of $10^{-5}$ and 50 bins in the phase space $[0,1.0]$. The PDM analysis yields the first guessed frequency $\left(f_{\mathrm{PDM}}\right)$ at the minimum $\Theta_{\mathrm{PDM}}$. With this frequency, the folded phase light curve is plotted and checked by eyes to exclude the harmonics, often the double or triple, which results in a crude estimation of the main frequency, $f_{\text {est }}=f_{\mathrm{PDM}} * n$ $(n=1,2,3 \ldots$ according to the order of the harmonics in the phased light-curves). In the second step, the frequency resolution is increased to $10^{-7}$ and the range of frequency is shrunk to $\left[f_{\text {est }}-0.05, f_{\text {est }}+0.05\right]$. Then a PDM analysis is performed once more to yield the minimum $\Theta_{\mathrm{PDM}}$ that tells the frequency with higher accuracy. Thanks to the long time span and numerous measurements of RRLS in the sample, such high precision is achievable in determining the frequency. This frequency is the main pulsation frequency, $f_{0}$ or corresponding period $P_{0}$ in following text, to distinguish among the fundamental, first overtone and second overtone modes.

Once the main frequency is determined, the light curve is fitted by a linear superposition of its harmonic oscillations:

$$
M(t)=M_{0}+\sum_{k=1}^{n}\left(a_{k} \sin k \omega t+b_{k} \cos k \omega t\right),
$$

where $n$ is the highest degree of the harmonics, $M(t)$ the measured magnitude in the I or $\mathrm{V}$ band, $M_{0}$ the mean magnitude, and $\omega=2 \pi / P_{0}$ the circular frequency. In fact, it's the phased light-curve instead of the light-curve itself that's fitted for the sake of higher significance:

$$
M(t)=M_{0}+\sum_{k=1}^{n}\left(a_{k} \sin 2 \pi k \Phi_{t}+b_{k} \cos 2 \pi k \Phi_{t}\right),
$$

where $\Phi_{t}=\left(t-t_{0}\right) / P-\left|\left(t-t_{0}\right) / P\right|, t$ is the time of observation and $t_{0}$ is the epoch of maximum brightness. Eq. (2) can be re-written as:

$$
M(t)=M_{0}+\sum_{k=1}^{n} A_{k} \sin \left(2 \pi k \Phi_{t}+\phi_{k}\right),
$$

where $A_{k}=\sqrt{a_{k}^{2}+b_{k}^{2}}$ and $\phi_{k}=\arctan \left(b_{k} / a_{k}\right)$. The parameters $A_{k}$ and $\phi_{k}$ can be transformed to the Fourier parameters $R_{i j}=A_{j} / A_{i}$ and $\phi_{i j}=j \phi_{i}-i \phi_{j}$ ( $i, j$ refers to different $k$, Simon \& Lee, 1981)), both of which are widely used in expressing the features of the light-curves, and even to derive the physical parameters of the variables such as the metallicity (e.g. Jurcsik \& Kovacs 1996).

In principle, the degree of the harmonics can be arbitrarily high, however, the highest degree in practice is set to 5 or 6 , being able to reflect the essential shape of the light curve. Among all the 655 stars, only 64 stars need the sixth harmonics and all the others with $n \leq 5$. Moreover, it avoids over-fitting, even for very complex light curves such as shown in Fig. 2 The main pulsation frequency we derived by this method is almost the same as that of Soszyński et al. (2009), with the difference $\leq$ 0.0001. The upper four panels in Fig. 2] show the process of determining the primary frequency, from the estimation of the frequency, through the accurate measurement of the frequency and the folded phase curve to the final fitting of the phase curve. 

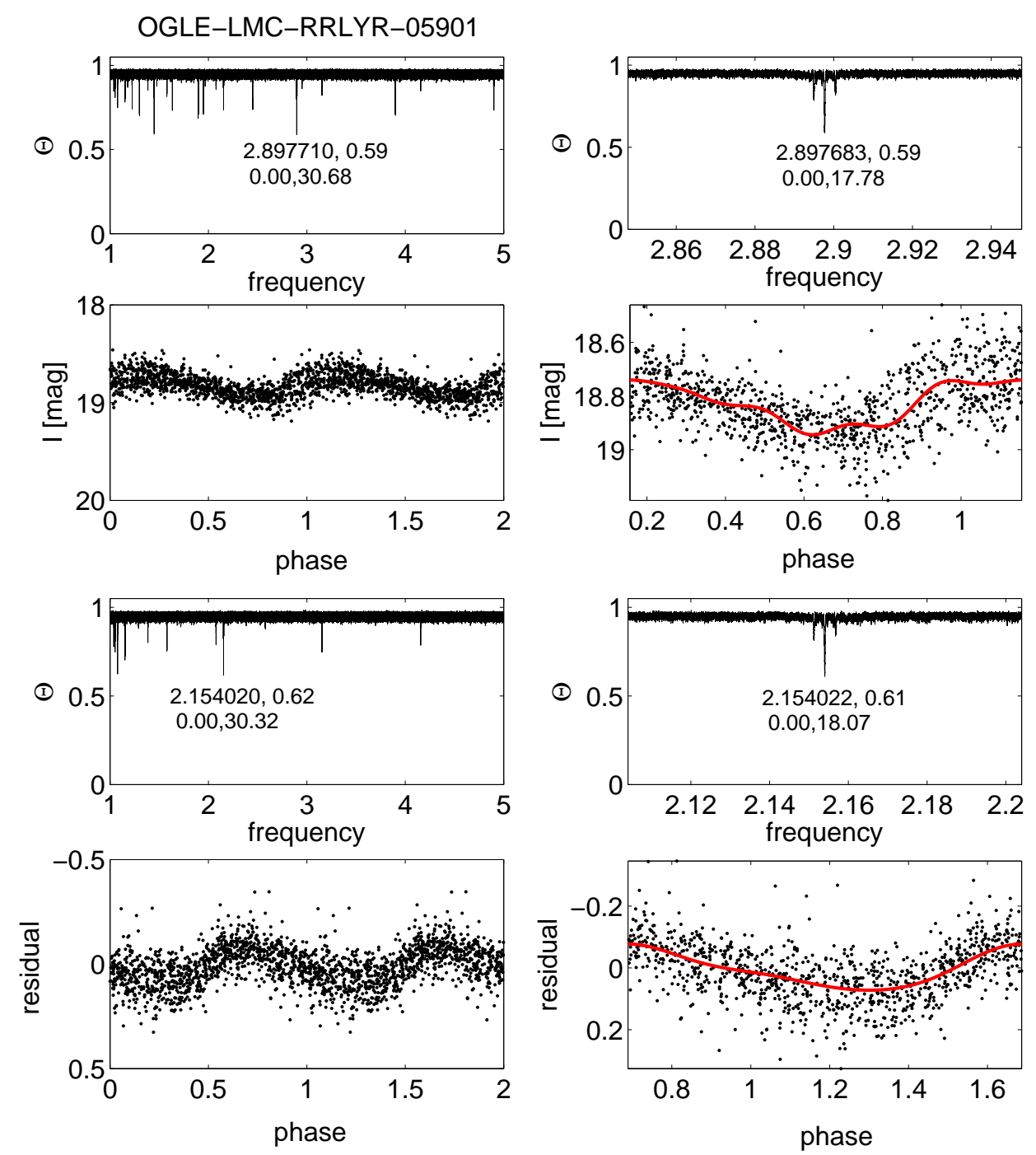

Fig. 2 An example of the frequency identification (for Star 3): the first loop to search for the primary frequency from original data (upper 4 figures) and the second loop to search for secondary period from the residual data after first prewhitening (lower four figures). For either loop of the four figures, the left two figures are $\Theta_{\mathrm{PDM}}-$ frequency diagram with frequency in $[0.2,1,0.00001]$, phased light-curve based on the frequency $f_{1 \text { st }}$, while the right two figures are $\Theta_{\mathrm{PDM}^{-}}$-frequency diagram with frequency in $\left[f_{\text {est }}-0.05, f_{\text {est }}+0.05,0.0000001\right]$, and phased light-curves based on the frequency $f_{\text {final }}$ where the red line is the Fourier fitting to the phased light-curve. The numbers shown in the $\Theta_{\mathrm{PDM}}$-frequency figure are the frequency derived and corresponding $\Theta_{\mathrm{PDM}}, P$ and $S / N$ described in Section 3. 
The secondary period is searched in the residual after subtracting the variation in the main frequency with its harmonics. The method is the same as for the principle period. The difference lies in the amplitude of the secondary oscillation being much smaller than the principle one. This procedure is carried repeatedly until an assigned threshold, which is set to guarantee the significance of the derived period. The phase dispersion parameter $\Theta$ is related to the significance of the period, the smaller the better. Another related parameter is the probability $P\left(F\left(P / 2, N-1, \sum\left(n_{j}\right)-M\right)=1 / \Theta\right)$ (Stellingwerf, 1978). However, $\Theta$ and $P$ depends on the number and quality of measurements so they do not necessarily have the same cut-off value in different cases and become ambiguous in marginal cases. We developed an independent parameter, $S / N \equiv \frac{\bar{\Theta}-\min (\Theta)}{\sigma_{\Theta}}$, which reflects the $\mathrm{S} / \mathrm{N}$ of the minimum $\Theta$ in the $\Theta$ distribution. Combining all the three parameters, the specific thresholds are set to $\Theta_{\mathrm{PDM}}<0.63-0.85$, $P<0.01-0.05$ or $S / N>10-15$ for a reliable period determination. Once the period is determined, a fitting is performed as for the principle period, but the highest degree of the harmonics is taken to be 3 instead of 6 in the first run. The lower four panels in Fig. 2 show the procedure for determining a secondary period, including the first guess and final determination of the frequency and the fitting of the phased light curve.

\section{CLASSIFICATION}

RRLS are considered as the pure radial pulsator basically. According to the pulsation modes, it was classically divided into four types: RRab pulsating in the fundamental (FU) mode, RRc in the first overtone (FO) mode, RRe in the second overtone mode (SO) and RRd in the double (FU and FO) modes. Alcock et al. (2000) introduced a new system of notation with a digit to mark the primary pulsation mode to replace the letters, i.e. RR0, RR1, RR2 and RR01 instead of RRab, RRc, RRe and RRd, more intuitive to mnemonics. When the modulation of period and amplitude is considered, Nagy \& Kovács (2006) adopted additional letters to classify RRLS, PC for period change, BL for the Blazhko effect and $\mathrm{MC}$ for closely spaced multiple frequency components. To make a complete view of the variation type, we combine both notations into a more detailed designation to make the phenomenological classification of RRLS by following Nagy \& Kovács (2006).

The identification of the main pulsation mode is clear for separating the RR0 and RR1 classes, as proved in many previous studies. The period is longer and the amplitude is mostly larger in RR0 RRLS than in RR1, and the shape of light curve of RR0-type RRLS is more asymmetric than the RR1type, as shown in the period-amplitude and period-skewness diagrams in Fig. 3 where the skewness is calculated from the phased light curve. The definition of skewness is $E(x-\mu)^{3} / \sigma^{3}$, where $\mathrm{x}$ is the observed magnitude, $\mu$ the mean of $\mathrm{x}, \sigma$ the standard deviation of $\mathrm{x}$, and $E(t)$ represents the expected value of the quantity $t$. Examples of phased light-curves in our sample are shown in Fig. 5, The gap between RR0 and RR1 is also clearly shown in the period-Fourier coefficients diagram in Fig. 4

The puzzle comes with the identification of the RR2-type RRLS, those pulsating in the secondovertone mode. The RR2 stars are believed to have even shorter period, slightly smaller amplitude and more symmetric light curve. In the period-amplitude diagram, they should locate on the left of RR1 stars, e.g. the magenta points in Fig.2 of Soszyński et al. (2009). In the period-amplitude diagram of our sample (Fig. 3 top), no clear gap is found in the shorter-period group of stars. There is neither apparent peak in the period distribution of all single-mode RRLS shown in Fig.6, which is very similar to that of all the RRLS in LMC (Soszyński et al., 2009). Concerning the shape of the light curves, their skewness is calculated. From the appearance in the bottom panel of Fig. 3, the separation between RR0 and RR1 is again apparent with RR1 being systematically more symmetric, while no more subgroup can be further distinguished in the relatively symmetric group. Thus, if a RR2 group is to be assigned, the borderline would be very arbitrary both in the period-amplitude and period-skewness diagrams. Because there is no systematic features, we are conserved in identifying a group of RR2 stars. In fact, it's also possible that the stars with shorter period, smaller amplitude and more sinusoidal light curve may be metal-rich RR1 stars (Bono et al., 1997a). 


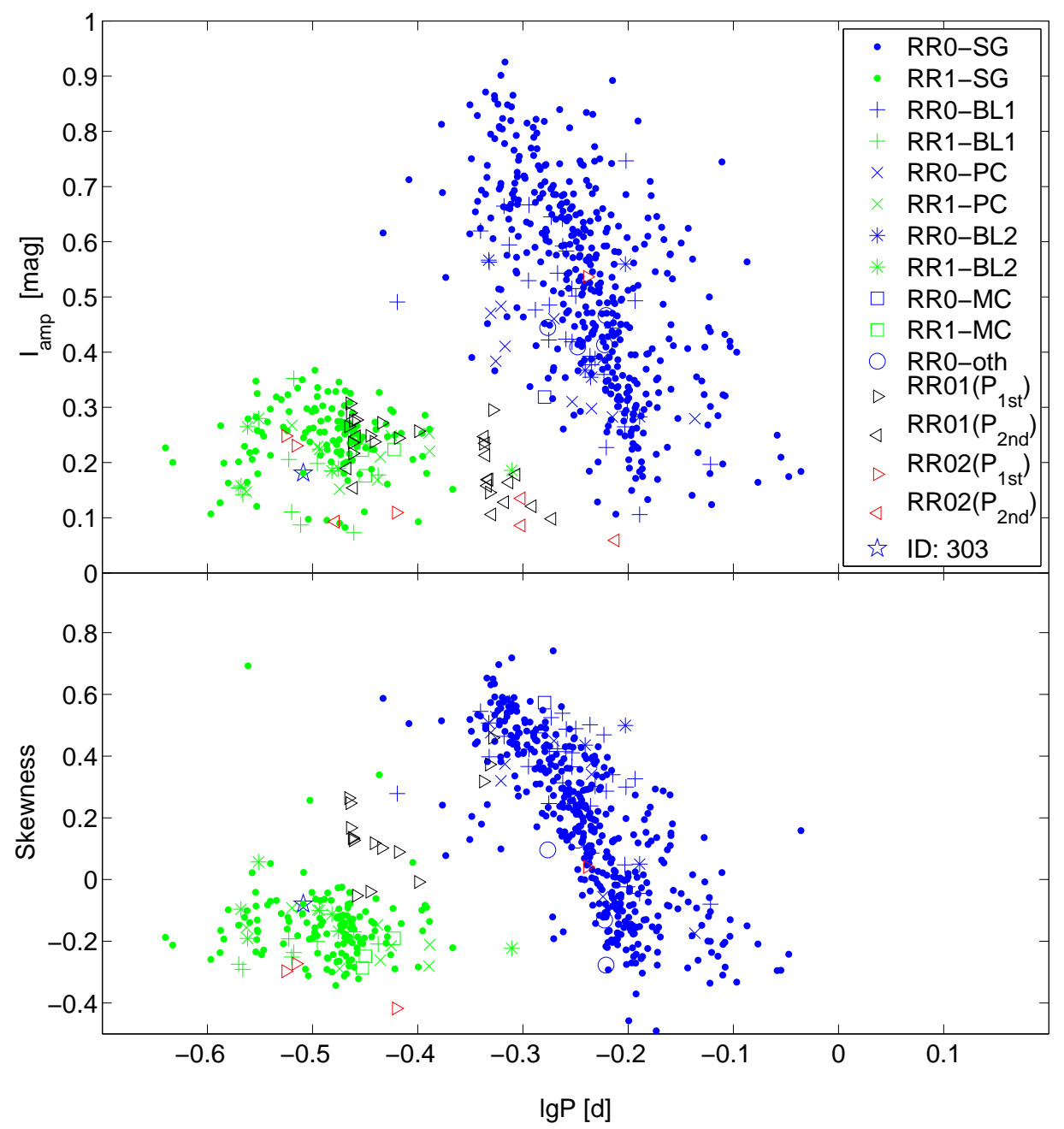

Fig. 3 The period-amplitude and period-skewness diagrams for all RRLS in our sample. The definition of skewness is given in the text. The meanings of the symbols are shown in the legend panel where $\mathrm{P}_{1 \text { st }}$ and $\mathrm{P}_{2 \text { nd }}$ means the primary and secondary period respectively in double-mode RRLS, and the meaning of specific classifications is described in text in Section 4.

\subsection{Single period RRLS}

The notation "RR-SG" refers to the single period RRLS, i.e. no more frequency is found in the residual after removing the primary frequency and its harmonics. In our sample, 556 stars were found to be RR-SG stars, $84.9 \%$ of all the 655 sample RRLS. Out of these RR-SG stars, $424(76 \%)$ are RR0-SG in the FU mode and $132(24 \%)$ are RR1-SG RRLS in the FO mode. The RR0-SG RRLS are three times as many as RR1-SG RRLS. In Table A.1 we listed their period of light variation, minimum phase 


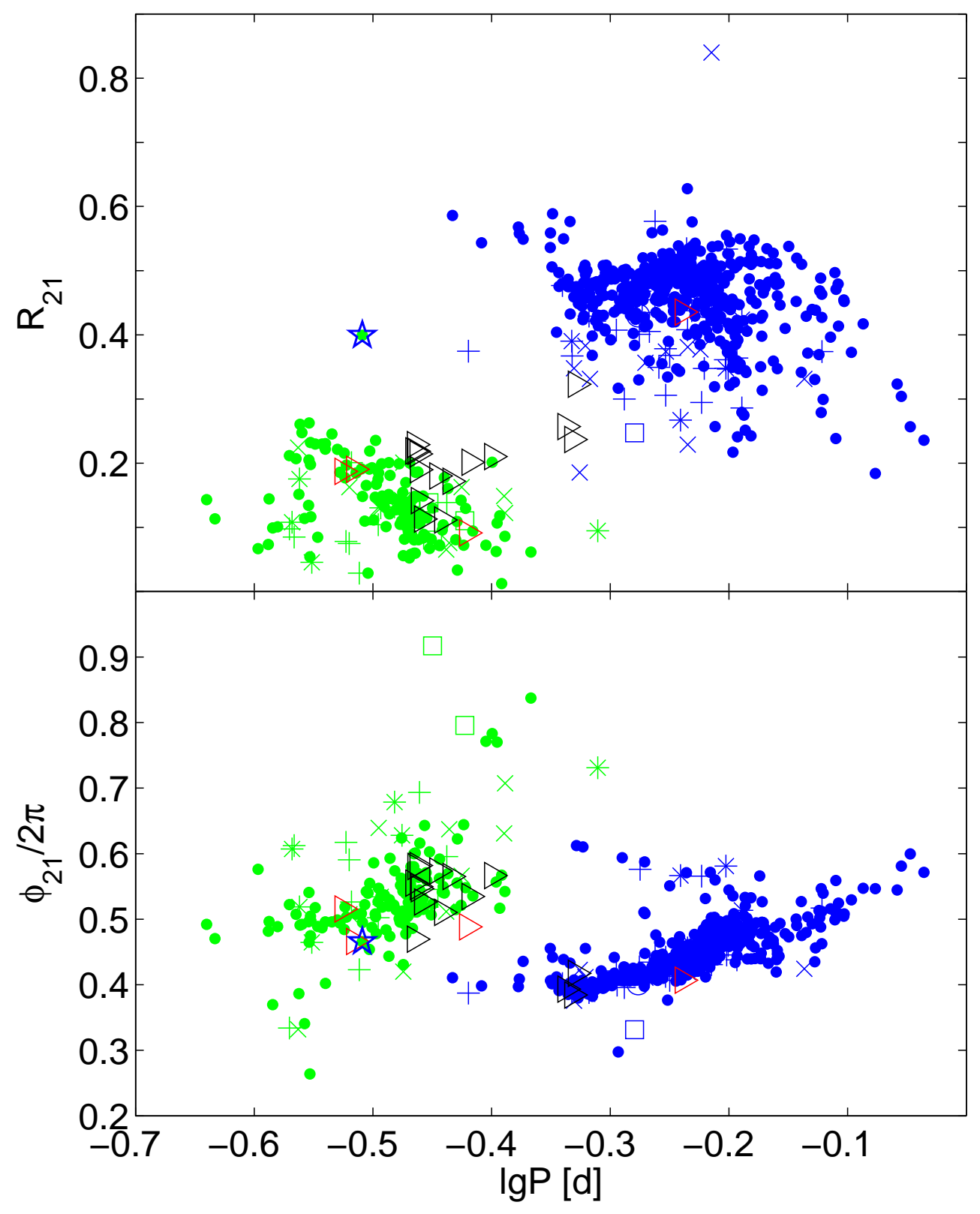

Fig. 4 The period-Fourier coefficients diagram. The symbols obey the same legend as in Fig. 3. The Fourier coefficients are defined in Eq.(3) with $\phi$ divided by $2 \pi$. 
dispersion $\Theta$, amplitude and mean magnitude in the I band, and subtype. The histogram of the RRLS periods (Fig. 6) displays two classical prominent peaks at $0.58 \mathrm{~d}$ and $0.34 \mathrm{~d}$ for RR0 and RR 1 stars respectively. The average period of our sample for RR0 stars $\bar{P}_{\mathrm{RR} 0-\mathrm{SG}}$ is $0.587 \mathrm{~d}$ and for RR1 stars $\bar{P}_{\mathrm{RR} 1-\mathrm{SG}}$ is $0.328 \mathrm{~d}$.

We compare our division of the subtype into RR0 and RR1 with that of Soszyński et al. (2009), regardless of RR2 stars. One star (ID: 303, OGLE ID: OGLE-LMC-RRLYR-14697) is found to be discrepant, which is classified as RR1 in our work and RR0 in their work. In Fig. 3 that is the main criteria for classification, this star is specially denoted by a pentagon. In either the period-amplitude or the period-skewness diagram, this star locates in the central area of RR1 stars. When compared with the RR0 and RR1 stars in the phased light curve (Fig. 5), its shape is apparently asymmetric, but not the same as RR0 stars (not so steep as RR0 stars). On the other hand, its small amplitude and short period bring it to the RR1 group. This example also tells that the skewness of the light curve of one class covers a wide range, and makes the separation of RR2 stars hard.

The distribution of RR-SG in Fig. 3 is composed of two typical parts, one sequence of RR0-SG and the other shape of bell for RR1-SG. The distribution of RR0-SG seems to be composed of two parts, one densely clumped on the left forms a line shape, the other loosely distributed on the right. This shape of distribution reminds one of the dichotomy into Oosterhoff I and II classes of Galactic RRLS due to different evolving phases. Such dichotomy was found in the Galactic fundamental-mode RRLS (Szczygieł et al., 2009). Fig. 7 compares the Bailey diagram of RR0-SG stars in LMC with that in the Galactic bulge (Collinge et al., 2006) and the Galactic field (Szczygieł et al., 2009), where the contours are for the density of RR0-SG stars, the solid and dashed lines are from Equation 2, 3, 4 and 5 of Szczygieł et al. (2009) for their fitting to the Oo I (left), Oo II (right) groups and their borderlines respectively in the Galactic field RRLS. It can be seen that the RR0 stars in LMC can be divided into the Oo I and Oo II groups as well as in the Galactic field and bulge. Although the two groups are not fitted, the solid and dash lines from Szczygieł et al. (2009) generally agree with the distribution. Moreover, the Oo I group RR0 stars are clearly dominating over the Oo II stars. This is consistent with the fact that the average period $\bar{P}_{\mathrm{RR} 0-\mathrm{SG}}=0.587 \mathrm{~d}$ is more approximate to $0.549 \mathrm{~d}$ of the average Oo I clusters than to $0.647 \mathrm{~d}$ of the average Oo II clusters (Clement \& Rowe, 2000; van Agt \& Oosterhoff, 1959). According to the contour diagram, the Oo I group RR0 in LMC stars have a slightly longer period $(\triangle \lg P \sim 0.02)$ than those in the bulge. If the bulge and field appearance in the contour diagram is taken into account, the Oo I distribution forms a series from the bulge to the field and then to the LMC from left to right, i.e. the period from short to long. This sequence coincides with that of the average metallicity in these three environments. Since metallicity influences the opacity and the mechanism for the light variation of RRLS is the $\kappa$ mechanism, the shift of the Oo I group may be caused by the metallicity difference. Indeed, the metal abundance also influences the distribution of OoI and Oo II RR0 stars in the Galactic field (Szczygieł et al., 2009).

\subsection{Multiple period RRLS}

There are $99(15.1 \%)$ RRLS with variation detected in the residual of the light curve after removing the principle frequency and its harmonics. They are further classified into several subclasses according to the number of additional frequencies and their locations relative to the main frequency, including the RR01, RR-BL, RR-MC, RR-PC and miscellaneous subtypes.

\subsubsection{RR01 stars}

RR01 refers to the RR Lyrae stars pulsating in double radial modes, one is the FU mode and the other is the FO mode, classically RRd stars. In our sample, 15 (2.3\% in the sample) stars are classified as RR01 stars. Seven of them have two frequencies detected. Other eight stars have three frequencies detected, but the third frequency is either the sum or the difference of the first and second frequency and thus dependent. All these 15 stars are listed in Table A.2. with the period and amplitude in the FU mode $\mathrm{P}_{0}$ and $\mathrm{A}_{0}$, the minimum phase dispersion $\Theta_{0}$ in deriving the FU mode, the mean magnitude in the I band, 

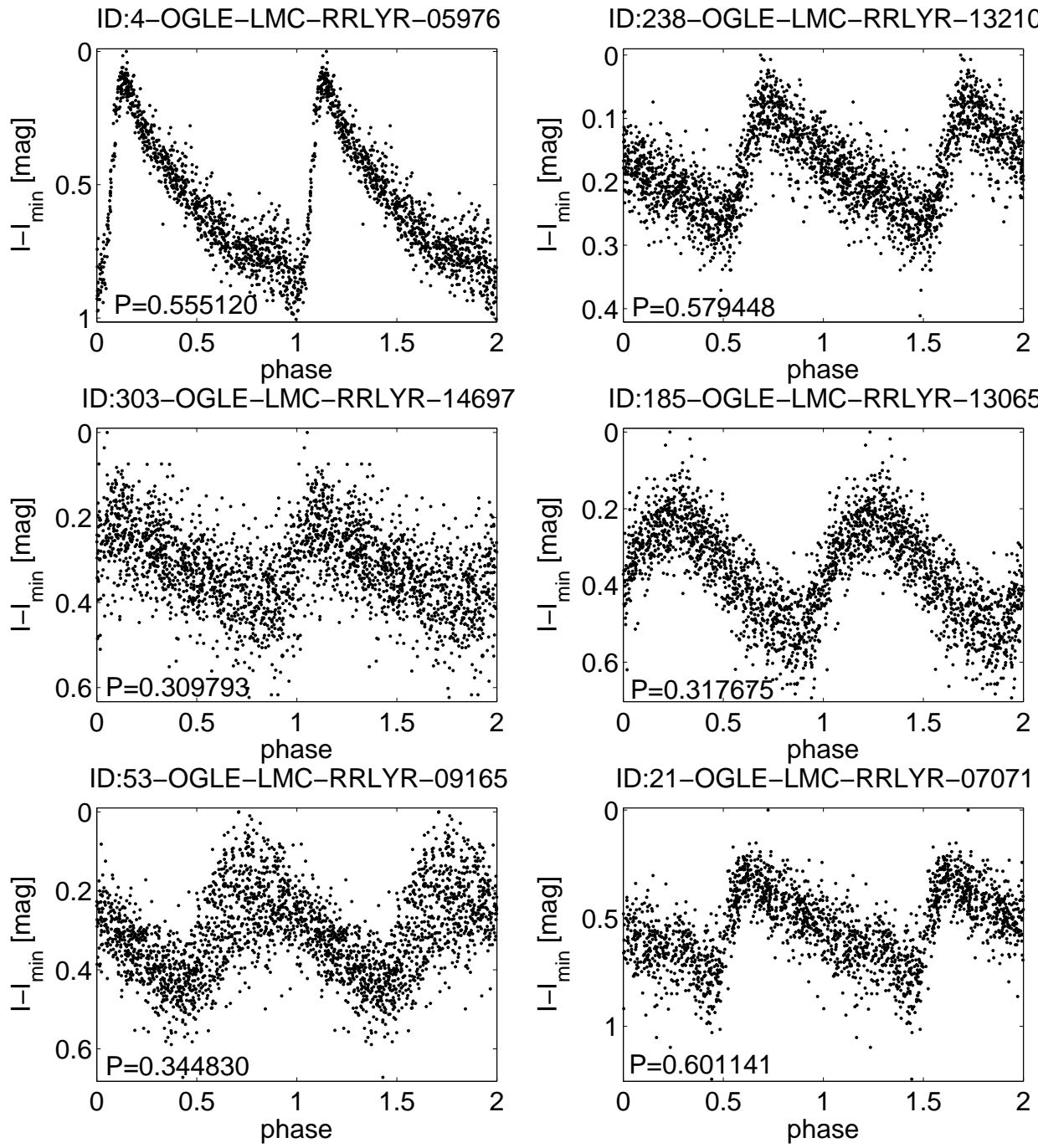

Fig. 5 Examples of the phased light-curves, including the controversial star ID303 (middleleft). The upper two stars are of type RR0, the middle two of RR1 and the lower two of RR01 (left) and RR02 (right) respectively.

the period and amplitude ratios between FO and FU modes, and the minimum phase dispersion $\Theta_{1}$ in deriving the FO frequency.

In the period-magnitude diagram (Fig. 3, top), both the FU and FO periods of these double mode RRLS are shown by black open triangles. The periods are either at the long side of the single FO mode or the short side of the FU RRLS. Their amplitudes are small, all smaller than 0.3 mag, which is comparable to that of the RR1 stars. The dominance of FO mode in RR01 can explain this small amplitude. It can be seen that the amplitude of the FU mode of RR01 stars is much smaller than those 


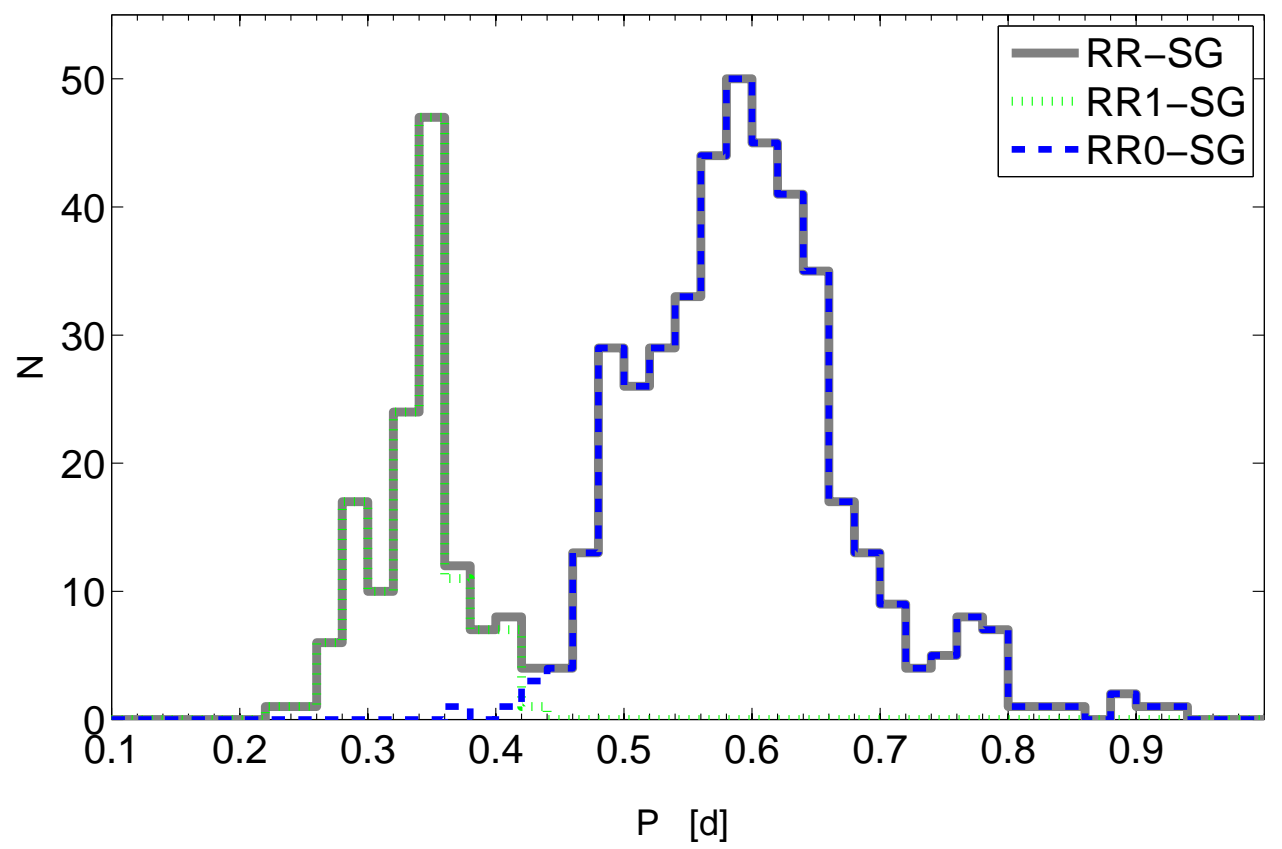

Fig. 6 Period distribution of all the single-mode RR-SG stars, with blue dash line for RR0SG, green dash line for RR1-SG, and gray solid line for the sum of RR0-SG and RR1-SG RRLS.

single mode FU stars at corresponding short period while comparable to those single mode FO stars. In fact, RR01 stars distinguish themselves from the other single-mode FU stars by their small amplitude in the period-amplitude diagram. Meanwhile, their light curves appear differently by a positive skewness from the FO RRLS most of which have a negative skewness although both are more symmetric than the FU RRLS. The lower panel of Fig. 3 shows such difference.

The period ratio $P_{1} / P_{0}$ ranges from 0.7422 to 0.7465 , with an average of 0.7436 . The amplitude ratio $A_{1} / A_{0}$ is significantly larger than one in 13 stars, and two smaller than one (but bigger than 0.8 ), with an average of 1.533 . It can be concluded that the RR01 stars mainly pulsate in the FO mode. In the Petersen diagram for RRLS (Fig. 8), the period and amplitude ratios are plotted versus the FU period, and compared with the results of Alcock et al. (2000) from the MACHO data. The increase of both ratios with the period is clear and consistent with Alcock et al. (2000). The distribution of the period ratio overlaps completely with that of Alcock et al. (2000). The ratio of the amplitude does not rise so high as the Alcock et al. (2000) result, although the rising tendency with period is the same. Moreover, the RR01 stars whose dominating mode is fundamental (i.e. $A_{1} / A_{0}<1$ ) have smaller $P_{1} / P_{0}$ than the average, specifically, their $P_{1} / P_{0}$ is all smaller than 0.743 , even when including those from Alcock et al. (2000).

\subsubsection{RR-BL stars}

RR-BL stars refer to the Blazhko stars. As mentioned in Introduction, the early identification of RR-BL stars was the symmetric appearance of the frequency spectrum. With the development of the study of the Balzhko effect, some asymmetric patterns are considered to be its variation. Thus, in the frequency 


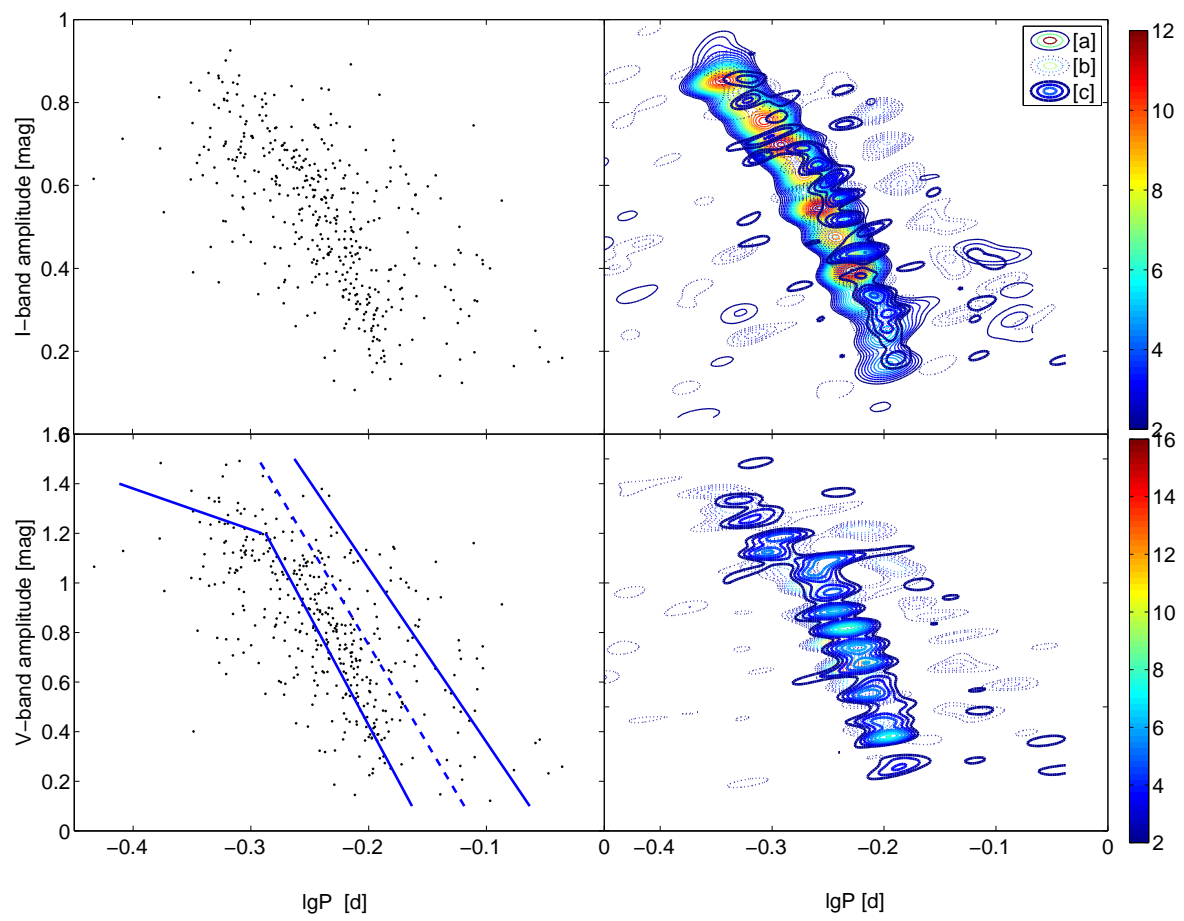

Fig. 7 Period-amplitude (I-band and V-band) diagram of RR0-SG stars. Contours show the distribution of the bulge RR0-SG stars from [a]: Collinge et al. (2006) and [b]: Szczygieł et al. (2009) as well as [c] of the LMC RR0-SG stars in this work. The two solid lines in the V-band diagram are from Szczygieł et al. (2009), showing the fitting of the Bailey diagram for the Oo I and II RRLS respectively, while the dashed line is the border to separate the Oo I type and Oo II type RRLS in the Galactic RR0 stars.

pattern, they may appear as: (1) two frequencies which have one closely spaced frequency around the principle frequency (RR-BL1), (2) three frequencies which have two side frequencies closely and symmetrically distributed around the main frequency (RR-BL2), and (3) more than three frequencies which have multiple components at closely spaced frequencies (RR-MC). All of them are the consequence of the modulation of the amplitude and/or phase, which can be explained by the modulation of a single (sinusoidal or non-sinusoidal) oscillation (Szeidl \& Jurcsik, 2009; Benkő et al., 2011).

RR-BL1 stars RR-BL1 stars have one frequency close to the main frequency, bigger or smaller. Alcock et al. (2000) marked them as $\nu 1$, and here we use the definition of Nagy \& Kovács (2006) to mark them as BL1. There are 41 (6.3\% of all the 655 stars in the sample) such RR-BL1 stars, forming a much larger group than other multi-period RRLS. Taking into account the main pulsation mode, they are classified into 32 RR0-BL1 stars in the FU mode and 9 RR1-BL1 stars in the FO mode. The main pulsation period of RR0-BL1 stars ranges from $0.38 \mathrm{~d}$ to $0.76 \mathrm{~d}$ and of RR1-BL1 from $0.26 \mathrm{~d}$ to $0.37 \mathrm{~d}$. The main pulsation amplitude of RR0-BL1 stars ranges from 0.106 mag to 0.747 mag and of RR1-BL1 from 0.073 mag to $0.352 \mathrm{mag}$. In Fig. 3 and Fig. 4 these RR-BL1 stars are mixed homogeneously with other single-mode RRLS, which means they have normal main period and amplitude of light variation as those single-mode stars. 


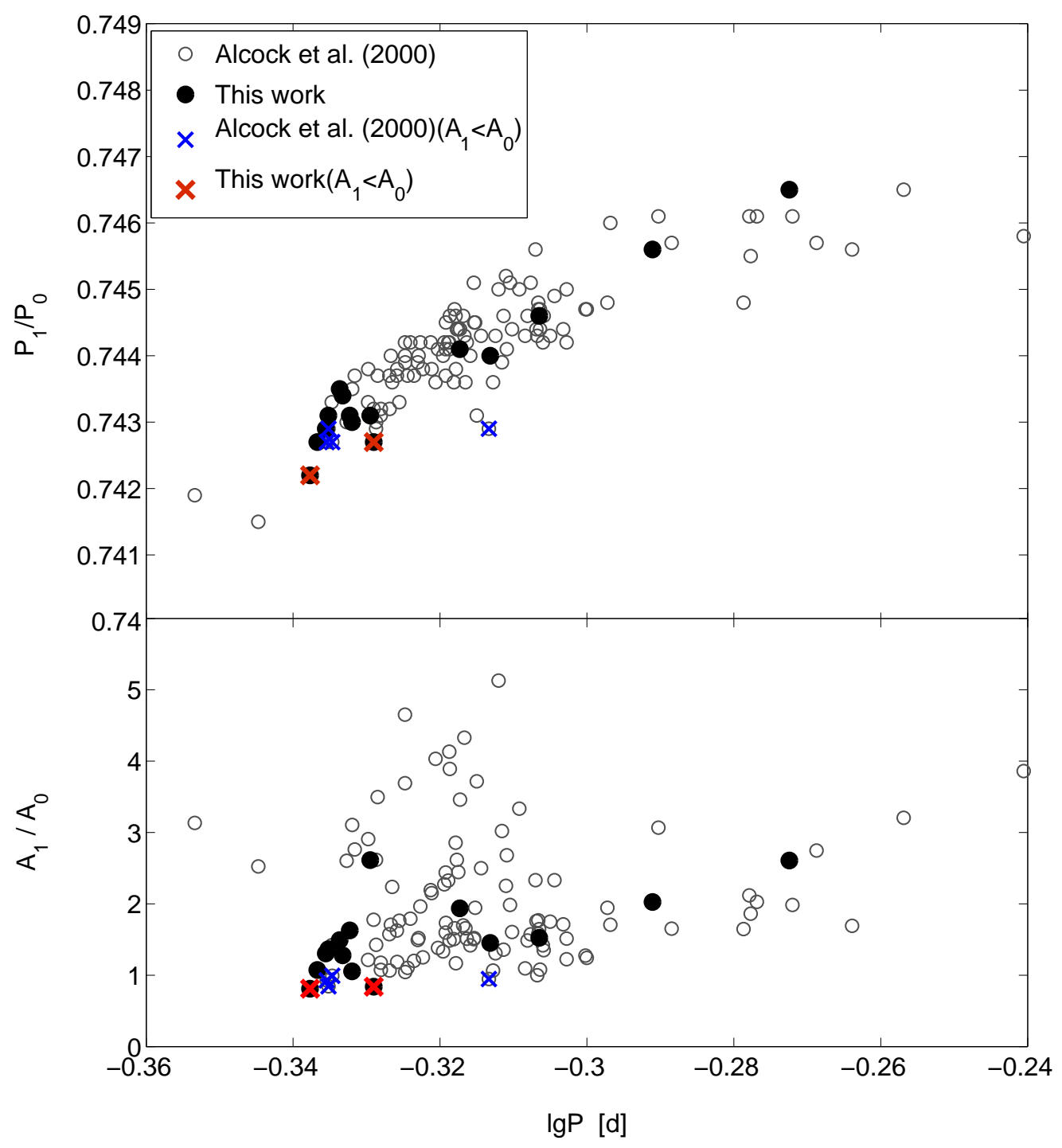

Fig. 8 Petersen's diagram for the RR01 stars (upper) and the amplitude ratio (lower) vs. $\lg \mathrm{P}$ diagram in LMC. The solid symbols are from our results while the hollow ones from the LMC data by the MACHO project (Alcock et al., 2000).

The sum of all the differences between every side frequency and main frequency $\delta f=\sum_{i} \Delta f_{i}$, where $\Delta f_{i}=f_{i}-f_{0}$ and $i=1,2 \ldots$ for frequency at the first, second overtone and so on, is usually used to characterize the asymmetry of the frequency distribution. For RR-BL1 stars, there is only one side frequency, resulting $\delta f=f_{1}-f_{0}$. About $75 \%$ (24 out of 32) of these RR0-BL1 stars and $67 \%$ (6 out of 9) of these RR1-BL1 stars have $\delta f$ positive. This is very different from the $37 \%$ proportion 
for RR1-BL1 stars derived from the MACHO data by Alcock et al. (2000). But it agrees well with the percentage $80 \%$ for RR0-BL1 stars from the study of the Blazhko variables by Kovács (2002).

For RR0-BL1 stars, the Blazhko periods vary between about 23 days and over 1500 days with an average of about 180 days and rms of 348 days. For RR1-BL1 stars, the Blazhko periods vary from 6.4 days to about 3000 days with an average of 745 days and rms of 1404 . The shortest modulation period (6.4 d) is comparable to that found for RR1-BL in LMC by Nagy \& Kovács (2006) and also consistent with those in the Galactic field for RR0-BL stars (Jurcsik et al., 2005b), i.e. around 6 days which is about 20 times of the main pulsation period. On the other end, the longest modulation period $\sim 3000 \mathrm{~d}$ is comparable to the time span of the data available, it is at least partly limited by the observational time coverage. With the continuation of the OGLE project, longer modulation period can be expected. The distribution of the Blazhko periods of RR-BL1 stars is shown in Fig. 10. The RR0-BL1 stars exhibit a normal distribution with the peak around 60-70 days. The RR1-BL1 stars shows a quite scattering distribution in a much wider range, although the group of only 9 RR1-BL1 objects makes this statistical significance less convincible. It seems that there is a preferred range of Blazhko period for RR0-BL1 from $32 \mathrm{~d}$ to $200 \mathrm{~d}$ (lgP from 1.5 to 2.3 ), but no such preferred range for RR1-BL1, which agrees well with the result of Nagy \& Kovács (2006).

We listed the main frequency and other variation parameters of RR-BL1 stars in Table A.3. The amplitude ratio $\left(A_{1} / A_{0}\right)$ of RR0-BL1 varies from 0.119 to 0.382 with an average of 0.237 and of RR1BL1 varies from 0.324 to 1.081 with an average of 0.574 . RR1-BL1 have a larger amplitude ratio than RR0-BL1, with one star (ID: 126) even larger than one but its main amplitude is small, only $0.087 \mathrm{mag}$.

The asymmetric frequency can be regarded as the extreme case of the amplitude asymmetry in the Blazhko effect when the invisible symmetric component is completely submersed in the noise. In Fig. 9 is shown an example of such situation (star ID: 78, OGLE ID: OGLE-LMC-RRLYR-09295). The upper two figures are the frequency- $\Theta_{\mathrm{PDM}}$ diagram and the phased light-curves of the first-loop period searching for the main frequency 1.7927. The lower left figure shows the successful search for the secondary frequency at 1.8108 , and the lower right figure shows that no more reliable frequency can be derived since all the three parameters at $f=1.7746\left(\Theta_{\mathrm{PDM}}=0.86\right.$, sig. $=0.015$ and $\left.\mathrm{S} / \mathrm{N}=8.7\right)$ are below our threshold. On the other hand, it may be expected that this frequency would be detected given a higher sensitivity of observation or a lower threshold. This example further supports that the missing of another frequency component in RR1 stars be caused by the asymmetry of the modulated amplitude.

RR-BL2 Stars For RR-BL2 stars, the secondary frequencies indicate the modulation of the amplitude and phase. There are $11(1.7 \%)$ RR-BL2 stars and they can be divided into two subtypes, 4 RR0-BL2 and 7 RR1-BL2 based on the main pulsation mode. This percentage $(1.7 \%)$ is much smaller than the RR-BL1 stars (6.3\%). It is also low in comparison with the results of previous studies, which will be discussed later. We think such low percentage is mainly due to our very strict criteria to identify a frequency so that some third frequencies have been dropped like the case of Star 78 shown in Fig. 9 This $1.7 \%$ percentage should be taken as the lower limit of the percentage of RR-BL2 stars.

The differences between the side and main frequencies are shown in Table A.4 i.e. $\triangle f_{+}$and $\triangle f_{-}$. They are both smaller than 0.1 . In addition, the difference between $\triangle f_{+}$and $\triangle f_{-}$is all smaller than 0.0003. It's these two features that bring them into the RR-BL2 class. On the ratio of the two amplitudes $A_{+}$and $A_{-}\left(A_{+} / A_{-}\right)$, it changes from 0.76 to 1.60 . This range of ratio means the two components have pulsation amplitudes at the same order, or we are only sensitive to such situation. This is understandable since a large ratio of the two amplitudes would surely make the weak component invisible and move the star into the RR-BL1 group. Such bias can only be alleviated by a very-high-sensitivity observation. This fact can also account for the low percentage of the BL2 stars.

As shown in Fig. 3 by the symbol asterisks, the RR-BL2 stars have ordinary period and amplitude in the principle pulsation mode. The amplitude ranges from 0.283 mag to 0.567 mag and the period from $0.465 \mathrm{~d}$ to $0.647 \mathrm{~d}$, with the average $\overline{A_{0}}=0.44 \mathrm{mag}$ and $\overline{P_{0}}=0.58 \mathrm{~d}$ for RR0-BL2. For RR1-BL2 stars, the amplitude ranges from $0.158 \mathrm{mag}$ to 0.265 mag and the period from $0.270 \mathrm{~d}$ to $0.489 \mathrm{~d}$, with the average $\overline{A_{0}}=0.21 \mathrm{mag}$ and $\overline{P_{0}}=0.33 \mathrm{~d}$. 

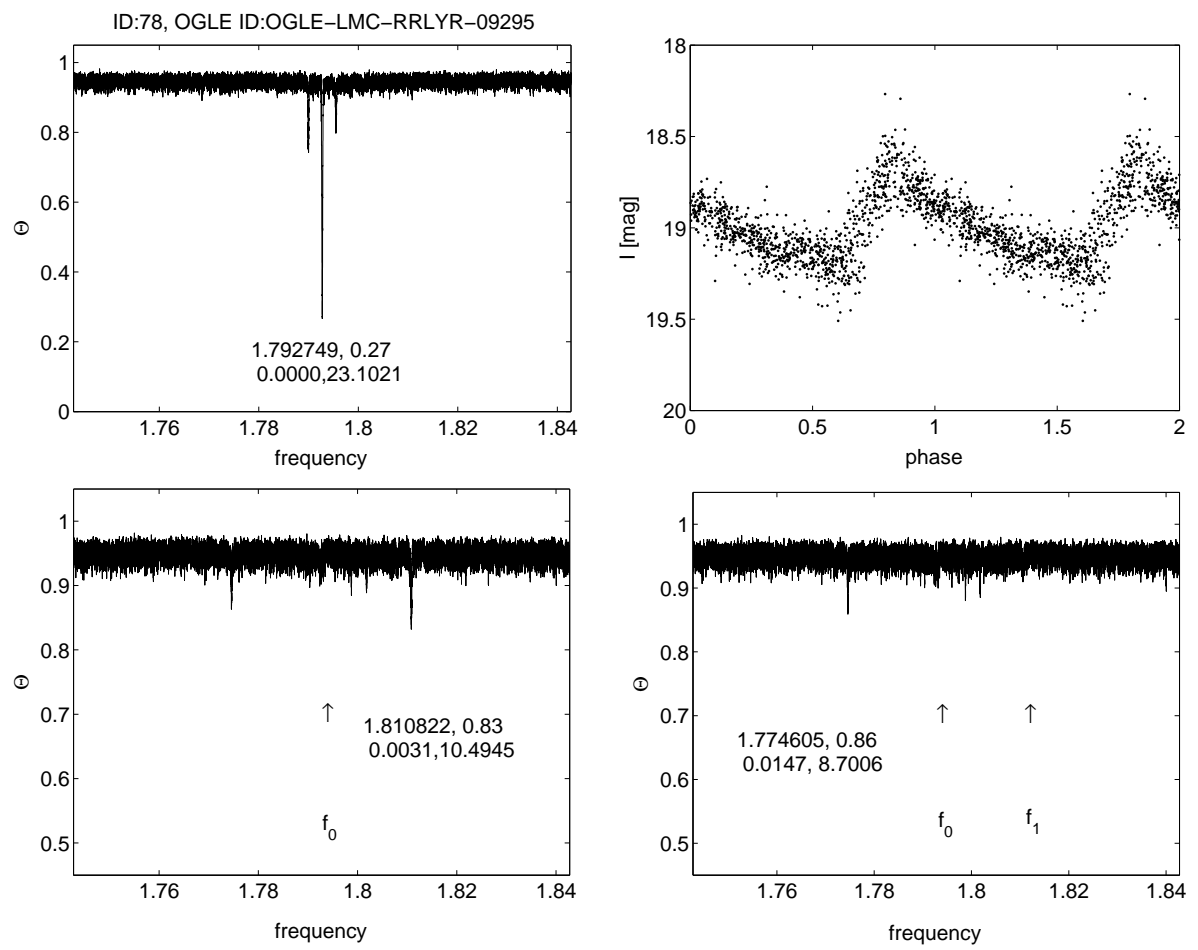

Fig. 9 One example (Star 78) of the BL1 star which shows asymmetric frequency as the result of the extreme amplitude asymmetry in the Blazhko effect when the invisible component is completely submersed in the noise, where the legends are the same as in Fig. 2 Notice that the $\Theta$-Frequency diagram is different from the frequency spectrum, the "triplets" shown in the first figure are only the main frequency and two aliases.

According to $\triangle f_{+}$and $\triangle f_{-}$, the modulation period varies from about 43 to over 2700 days with an average of 1349 days for RR0-BL2; and from 12 to 2902 days with an average of 1288.5 days for RR1-BL2. The distribution of the modulation frequencies are shown in Fig. 10, Because the volume of the RR-BL2 stars is small, the distribution does not exhibit any outstanding feature. However, the situation becomes clearer when the RR-BL1 stars are included, which is reasonable since BL1 stars can be regarded as the extreme case of RR2 and both are Blazhko variables. Consequently, our sample of 655 RRLS contains 52 Blazhko stars. In Fig. 10, the period distribution of all the RR0-BL and RR1-BL stars is shown. Because of the dominance of RR-BL1 stars, the distribution of RR-BL stars is similar to that of RR-BL1 stars, i.e. with a preferred range of period from a few tens to a couple of hundred days. In regards to the modulation amplitude, a correlation is found with the main pulsation amplitude. As shown in Fig. 10 (bottom), a linear fitting results in that $A_{i}=0.106 * A_{0}+0.057$ and the correlation coefficient is 0.605 which means significant correlation. The error here we adopted is the maximum of the photometric error assigned in the catalog which is apparently bigger than the error in the fitting. The order of the error is mostly around $0.1 \mathrm{mag}$. This correlation was not found before and neither predicted in any models for the Blazhko effect. But it indicates that the Blazhko modulation is related to the main pulsation mode and it should be taken into account in models. On the contrary, Jurcsik et al. (2005a) found that the possible largest value of the modulation amplitude, defined as the sum of the 

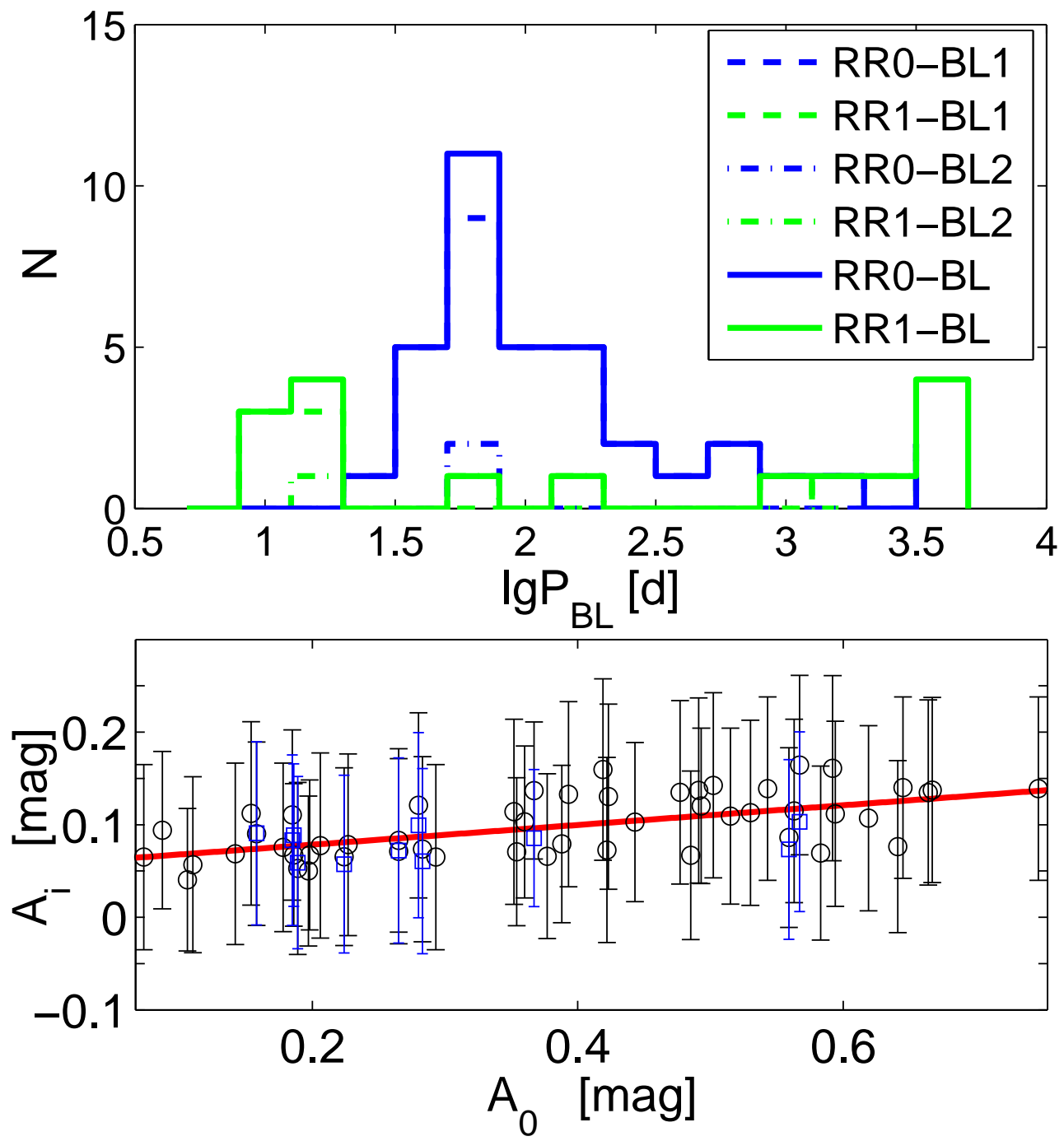

Fig. 10 Top: distribution of $\lg P_{\mathrm{BL}}\left(P_{\mathrm{BL}}\right.$ : the Blazhko modulation period), where the meaning of symbols are explained in legends. Bottom: the modulation amplitude vs. the main pulsation amplitude, where black filled circles denote the amplitudes of the second (in case of RRBL2) component of modulation, and the black solid line is the linear fitting between the two parameters.

Fourier amplitudes of the first four modulation frequency components, increases towards shorter period variables.

RR-MC stars We have got 4 RR-MC in our sample. Actually all of them have three side frequency components. One RR-MC star is in the FU mode and three are in the FO mode. According to the structure of the side frequencies, they show three patterns, similar to the RR-MC stars described in Nagy \& Kovács (2006): (a) two of the three frequencies are symmetric to $f_{0}$; (b) none is symmetric to 
the others, but three frequencies are at both sides to $f_{0} ;(\mathrm{c})$ three side frequencies are all at one side to $f_{0}$. In Table A.5 the four RR-MC stars are shown with their patterns in the column "Notes". These stars with multiplets may also be Blazhko stars (Benkô et al., 2011).

\subsubsection{RR-PC stars}

RR-PC refers to period-changing stars. It's difficult to distinguish PC stars from MC stars or BL stars since they all have closely spaced side frequencies. Nagy \& Kovács (2006) defined the PC stars as that they have close components which can not be eliminated within three prewhitening cycles or their separation from the main pulsation component is $\leq \sim 1 / \mathrm{T}$, where $\mathrm{T}$ is total time span. The definition is followed in this work. In our sample, we find that no star has any frequency detected after four prewhitening loops, i.e. the number of frequencies are not bigger than 4 . The two stars which have a fourth frequency component both have at least one frequency with the separation from the main pulsation component $\leq \sim 1 /$ T. So they are classified as RR-PC stars with no doubt. In addition, there are some RRLS which have fewer than four frequency components but with some frequency whose separation from the main component is $\leq \sim 1 / \mathrm{T}$, the number of such stars is 18 . Altogether, there are $20 \mathrm{RR}-\mathrm{PC}$ stars, that is about 3 percent of the sample. Their variation properties are listed in Table A.6 Our attempt to analyze the period variation is hampered by the large interval between adjacent measurements which ranges from $0.003 \mathrm{~d}$ to $300 \mathrm{~d}$ with a mean value of about $3.6 \mathrm{~d}$, that is to say, the interval is several periods long.

\subsubsection{Other RRLS}

Eight $(1.2 \%)$ multi-period RRLS in our sample cannot be classified into the above subtypes. They have more than one pulsation frequency, but their frequencies are not closely spaced from the main frequency. Table 2 shows their frequencies and other variation parameters.

Four of them have one period around $0.3 \mathrm{~d}$ and the other around $0.5 \mathrm{~d}$, yielding a period ratio around 0.6 which is the canonical period ratio between the SO and FU modes (Bono et al., 1997b). So we suspect that although we can't find single SO mode pulsating RRLS, but they can co-exist with the FU mode. The four RR02 candidates are shown by red triangles in Fig. 3 where $\mathrm{P}_{1 \text { st }}$ and $\mathrm{P}_{2 \text { nd }}$ refer to the primary and secondary period respectively. As same as the RR01 stars, the interaction between the two modes have led their amplitude and period to be on the short/long end of the FU/SO mode, which bring them together in the period-amplitude diagram (Fig. 3). Moreover, Star 228 seems to have a long modulation period for its FU mode from the fact that a third frequency is found to be closely spaced to its FU frequency.

Only four stars were clearly claimed to be RR02 stars in the recent two years. All of them were discovered in space mission. They are V350 Lyr (Benkô et al., 2010) and KIC 7021124 (Nemec et al., 2011) from the Kepler mission, CoRoT 101128793 (Poretti et al., 2010) and V1127 Aql (Chadid et al., 2010) from the CoRoT mission. We found that star MACHO 18.2717.787 unidentified by Nagy \& Kovács (2006) from MACHO dataset could also be such double-mode RR02 star with the period ratio of 0.5810. Poretti et al. (2010) computed a grid of linear RRLS models in a large stellar parameter space which delineated a rough range of the RR02 stars in the Petersen diagram. A similar work was presented by Nemec et al. (2011) who used the Warsaw pulsation hydrocode including turbulent convection. In Fig. 11, the range of RR02 in the Petersen diagram defined by the two models are delimited by solid and dash lines respectively. It can be seen that the two models agree with each other generally but also disagree in particular at the short fundamental periods. In this diagram, Star MACHO 18.2717.787 denoted by a blue dot is definitely inside the model range. The four RR02 candidates from our sample are shown by red dots with other five such stars by dots in other colors. Star 159 and 535 are undoubtedly inside the range by both models. Star 228 is just outside the upper border of the models, but can not be excluded since the models surely have some uncertainty. The only star which apparently deviates from the models is Star 34 although it is not too far. Puzzlingly, all the nine stars take a trend that the period ratio increases with FU period, which is opposite to the models. Interestingly, such dis- 
Table 2 Parameters of light variation for miscellaneous RRLS.

\begin{tabular}{|c|c|c|c|c|c|c|c|c|}
\hline ID & $P_{0}$ & $P_{1}$ & $P_{2}$ & $A_{0}$ & $I$ & $P_{\text {short }} / P_{\text {long }}$ & $A_{\text {shortP }} / A_{\text {longP }}$ & Type \\
\hline 21 & 0.601141 & 0.997709 & & 0.466 & 19.032 & 0.6025 & 3.899 & RR0-D1 \\
\hline 34 & 0.379702 & 0.613512 & & 0.109 & 18.667 & 0.6189 & 1.846 & RR02 \\
\hline 159 & 0.576922 & 0.332432 & & 0.536 & 18.733 & 0.5762 & 0.185 & RR02 \\
\hline 181 & 0.564876 & 0.815054 & & 0.409 & 18.191 & 0.6931 & 4.964 & ? \\
\hline 228 & 0.304926 & 0.499391 & 0.499175 & 0.231 & 18.599 & 0.6106 & 1.712 & RR-02 \\
\hline 248 & 0.599241 & 0.464902 & & 0.413 & 18.806 & 0.7758 & 0.147 & ? \\
\hline 452 & 0.529150 & 0.997639 & & 0.445 & 18.483 & 0.5304 & 2.092 & RR0-D1 \\
\hline 535 & 0.298121 & 0.499435 & & 0.248 & 18.655 & 0.5969 & 2.879 & RR02 \\
\hline
\end{tabular}

crepancy between observation and model occurs exactly the same in RR01, the other double-mode stars (see Fig.5 of Alcock et al. 2000).

Two stars have a secondary period around 1 day. This frequency is not taken as the alias because the phased light-curves at this frequency show reliable periodicity. We hereby denote them as RR0D1, following Alcock et al. (2000). The other three stars can not be classified into any of the classes described above. We just mark them by "?" in Table 2

\section{DISCUSSION AND SUMMARY}

The incidence rates of each subclass are shown in Table 3 The majority, $85 \%$, is single-mode pulsators. The RRLS exhibiting the Blazhko effect (sum of RR-BL1 and RR-BL2) is the second most numerous group. With 52 RR-BL stars, they consist $7.9 \%$ of the sample. The incidence rates of Blazhko stars are compared with previous results in Table 4 . As our identification of a frequency is quite strict, the percentages in our sample should be taken as the lower limit of the Blazhko incidence rate.

For RRLS in LMC, the Blazhko variables (sum of RR-BL2 and RR-BL1 stars) occur less frequently in RR0 $(7.5 \%)$ than in RR1 stars $(9.1 \%)$. For RR1 stars, we can see an increasing trend of the incidence rates from $2.0 \%$ and $7.5 \%$ in previous work to $9.1 \%$ in present work, this can be explained by the longer time span and more precise data. But this trend does not show in RR0 stars although the data has been improved as for RR1 stars. To further analyze the reason, it is found that the incidence rate of RR-BL1 is comparable to previous work, the rate is $6.7 \%$ for RR0-BL1 and 5.1\% for RR1-BL1, compared to $6.5 \%$ (Alcock et al., 2003) and 3.5\% (Nagy \& Kovács, 2006). But in regarding to the RR-BL2 stars, the incidence rate of RR1-BL2 is comparable to the work before, with $5.1 \%$ to $3.5 \%$. Meanwhile the incidence rate of RR0-BL2 is abnormally low, with only $0.8 \%$ compared to $5.4 \%$ in Alcock et al. (2003). As mentioned in previous section, the reason may lie on our very strict criteria to accept a frequency which can move a BL2 star into a BL1 star in the case of high asymmetry of the amplitude in the two side frequencies. This explanation finds support in the fact that the RR0-BL2 stars have the amplitude ratio $A_{+} / A_{-}$not far from unity. Another possible reason is that, among RR0-BL1 stars, $75 \%$ (24 out of 32) have $f_{+}$, and all the four RR0-BL2 stars have $A_{+}>A_{-}$. In the work of Kovács (2002), 80\% of RR0-BL1 stars were found to have $A_{+}$component. We suspect that for RR0-BL stars, there maybe some unknown effect to make $A_{+}$much larger than $A_{-}$, which made a lot of $A_{-}$components missing. This effect does not appear in RR1 stars, for example, Alcock et al. (2000) found 37\% RR1-BL1 stars have $A_{-}$components, and in our sample only $43 \%$ RR1-BL2 stars have $A_{+}>A_{-}$. Based on Eq.(45) from Benkő et al. (2011), most of the RR0 stars have $\pi<\phi_{m}<2 \pi$; while for those RR1 stars, $\phi_{m}$ is evenly distributed between zero and $2 \pi$.

People used to believe that the incident rates of the Blazhko variables are lower in LMC than in the Galaxy. But this only suits for RR0 stars, may not be true for RR1, as the work of Nagy \& Kovács (2006) already suggested. From our work, with the long time span of observation, the Blazhko incidence rate for RR1 stars is larger in LMC than in the Galaxy bulge, as the rates are $5.1 \%$ and $4.0 \%$ for RR1-BL1 and RR1-BL2 respectively in our LMC sample in comparison with the $3.1 \%$ and $1.5 \%$ from Moskalik \& Poretti (2003) or 2.9\% and 3.9\% from Mizerski (2003) for the bulge RRLS sample. On the other hand, both the improvement of the observational precision and the extension of observational 


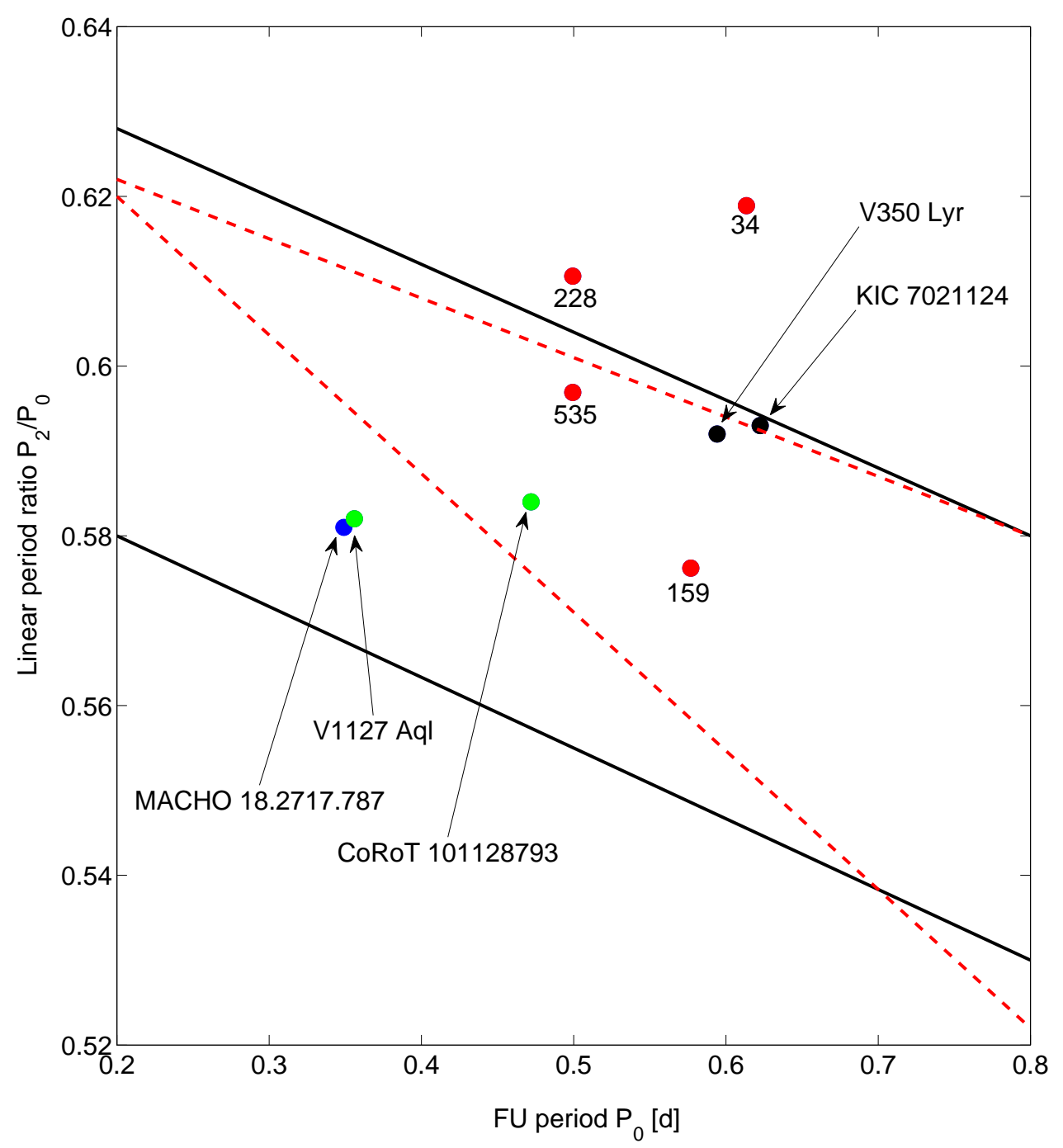

Fig. 11 The RR02 candidate stars in the Petersen diagram. The black solid lines delineate the rough range of RR02 stars by models from Poretti et al. (2010), and the red dash line from Nemec et al. (2011). The blue, green, black and red dots show the candidate RR02 stars from MACHO, CoRoT, Kepler datasets and our results from OGLE III datasets, with the name or ID number labeled (see Section 6.4 for details).

span increase the possibility to detect the Blazhko effect. Based on the data from the Kepler mission Kolenberg et al. (2010) and the Konkoly Blazhko Survey (Jurcsik et al., 2009), the incidence rate can exceed forty percent, but they are small samples with no more than 30 objects. Thus such comparison may not be conclusive as the observation and the analysis techniques are not uniform, and the samples are very different. According to these observations of LMC, SMC, bulge and $\omega$ Cen, there is no clear 
Table 3 Statistical results of the RRLS classification.

\begin{tabular}{ccrrrr}
\hline \hline type & Short description & number & percent & subtype & number \\
\hline RR-SG & Single-period & 556 & $84.9 \%$ & RR0-SG & 424 \\
& & & & RR1-SG & 132 \\
\hline RR01 & FU/FO double mode & 15 & $2.3 \%$ & & \\
\hline RR02 & FU/SO double mode & 4 & $0.6 \%$ & & \\
\hline RR-BL & One close component & 52 & $7.9 \%$ & RR0-BL1 & 32 \\
& & & & RR1-BL1 & 9 \\
& Two symmetric components & & & RR0-BL2 & 4 \\
& & & & RR1-BL2 & 7 \\
\hline RR-MC & Multiple close components & 4 & $0.6 \%$ & RR0-MC & 1 \\
& & & & RR1-MC & 3 \\
\hline RR-PC & Period changing & 20 & $3.1 \%$ & RR0-PC & 11 \\
& & & & RR1-PC & 9 \\
\hline RR-D1 & Second frequency at unity & 2 & $0.3 \%$ & RR0-D1 & 2 \\
\hline RR-? & Mysterious double mode & 2 & $0.3 \%$ & RR0-others & 2 \\
\hline
\end{tabular}

Table 4 The incidence rates of Blazhko effect in the sample compared to previous results. The references in the table are: (a): Moskalik \& Poretti (2003), (b): Alcock et al. (2003), (c): Mizerski (2003), (d): Collinge et al. (2006), (e): Moskalik \& Olech (2008), (f): Alcock et al. (2000), (g): Nagy \& Kovács (2006), (h): this work.

\begin{tabular}{crrrrrr}
\hline \hline Ref. & Bulge(a) & Bulge(c) & Bulge(d) & LMC(b) & $\omega$ Cen (e) & LMC(h) \\
\hline RR0 & 150 & 1942 & 1888 & 6391 & 70 & $\mathbf{4 7 8}$ \\
RR0-BL1 & $16.7 \%$ & $12.5 \%$ & $8.8 \%$ & $6.5 \%$ & $4.3 \%$ & $\mathbf{6 . 7 \%}$ \\
RR0-BL2 & $6.0 \%$ & $7.4 \%$ & $14.9 \%$ & $5.4 \%$ & $18.6 \%$ & $\mathbf{0 . 8 \%}$ \\
RR0-MC & & $4.4 \%$ & $3.5 \%$ & $0.3 \%$ & & $\mathbf{0 . 2 \%}$ \\
RR0-PC & & & $6.3 \%$ & $2.9 \%$ & & $\mathbf{2 . 3 \%}$ \\
\hline \hline Ref. & Bulge(a) & Bulge(c) & LMC(f) & LMC(g) & $\omega$ Cen (e) & LMC(h) \\
\hline RR1 & 65 & 771 & 1327 & 1332 & 81 & $\mathbf{1 7 7}$ \\
RR1-BL1 & $3.1 \%$ & $2.9 \%$ & $1.8 \%$ & $3.5 \%$ & $21.0 \%$ & $\mathbf{5 . 1 \%}$ \\
RR1-BL2 & $1.5 \%$ & $3.9 \%$ & $0.2 \%$ & $4.0 \%$ & $4.9 \%$ & $\mathbf{4 . 0 \%}$ \\
RR1-MC & & $5.3 \%$ & $0.4 \%$ & $1.0 \%$ & & $\mathbf{1 . 7 \%}$ \\
RR1-PC & & & $10.6 \%$ & $14.0 \%$ & & $\mathbf{4 . 5 \%}$ \\
\hline
\end{tabular}

relation between the incidence rate and metallicity. What causes the difference in the Blazhko incidence rate in different environments is unclear, which could be part of the difficulty in understanding the mechanism for the Blazhko effect.

Acknowledgements We sincerely thank the OGLE team for their continuing efforts and generosity in sharing data. We also thank the referee for his very helpful suggestions. This work is supported by the NSFC grant No. 10973004.

\section{References}

Alcock, C., Allsman, R., Alves, D. R., et al. 2000, ApJ, 542, 257

Alcock, C., Alves, D. R., Becker, A., et al. 2003, ApJ, 598, 597

Benkő, J. M., Kolenberg, K., Szabó, R., et al. 2010, MNRAS, 409, 1585

Benkő, J. M., Szabó, R., \& Paparó, M. 2011, MNRAS, 417, 974

Blažko, S. 1907, Astronomische Nachrichten, 175, 325

Bono, G., Caputo, F., Cassisi, S., Incerpi, R., \& Marconi, M. 1997a, ApJ, 483, 811

Bono, G., Caputo, F., Cassisi, S., et al. 1997b, ApJ, 477, 346

Borkowski, K. J. 1980, Acta Astronomica, 30, 393

Buchler, J. R., \& Kolláth, Z. 2011, ApJ, 731, 24

Chadid, M., Benkő, J. M., Szabó, R., et al. 2010, A\&A, 510, A39 
Clement, C. M., \& Rowe, J. F. 2000, in American Astronomical Society Meeting Abstracts \#196, Bulletin of the American Astronomical Society, vol. 32, 739

Collinge, M. J., Sumi, T., \& Fabrycky, D. 2006, ApJ, 651, 197

Dziembowski, W. A., \& Mizerski, T. 2004, Acta Astronomica, 54, 363

Feast, M. W., Abedigamba, O. P., \& Whitelock, P. A. 2010, MNRAS, 408, L76

Goupil, M., \& Buchler, J. R. 1994, A\&A, 291, 481

Jurcsik, J., \& Kovacs, G. 1996, A\&A, 312, 111

Jurcsik, J., Sódor, Á., Szeidl, B., et al. 2009, MNRAS, 400, 1006

Jurcsik, J., Sodor, A., \& Varadi, M. 2005a, Information Bulletin on Variable Stars, 5666, 1

Jurcsik, J., Szeidl, B., Nagy, A., \& Sodor, A. 2005b, Acta Astronomica, 55, 303

Kolenberg, K., Szabó, R., Kurtz, D. W., et al. 2010, ApJ, 713, L198

Kovács, G. 2002, in IAU Colloq. 185: Radial and Nonradial Pulsationsn as Probes of Stellar Physics, Astronomical Society of the Pacific Conference Series, vol. 259, edited by C. Aerts, T. R. Bedding, \& J. Christensen-Dalsgaard, 396-403

Kovács, G. 2009, in American Institute of Physics Conference Series, American Institute of Physics Conference Series, vol. 1170, edited by J. A. Guzik \& P. A. Bradley, 261-272

Li, Y., Wang, J., Wei, J.-Y., \& He, X.-T. 2011, Research in Astronomy and Astrophysics, 11, 833

Mathis, J. S. 1990, ARA\&A, 28, 37

Mizerski, T. 2003, Acta Astronomica, 53, 307

Montgomery, M. H., \& Odonoghue, D. 1999, Delta Scuti Star Newsletter, 13, 28

Moskalik, P., \& Olech, A. 2008, Communications in Asteroseismology, 157, 345

Moskalik, P., \& Poretti, E. 2003, A\&A, 398, 213

Nagy, A., \& Kovács, G. 2006, A\&A, 454, 257

Nemec, J. M., Smolec, R., Benkő, J. M., et al. 2011, MNRAS, 417, 1022

Pejcha, O., \& Stanek, K. Z. 2009, ApJ, 704, 1730

Pietrukowicz, P., Udalski, A., Soszynski, I., et al. 2011, ArXiv e-prints

Poretti, E., Paparó, M., Deleuil, M., et al. 2010, A\&A, 520, A108

Shibahashi, H. 2000, in IAU Colloq. 176: The Impact of Large-Scale Surveys on Pulsating Star Research, Astronomical Society of the Pacific Conference Series, vol. 203, edited by L. Szabados \& D. Kurtz, 299-306

Simon, N. R., \& Lee, A. S. 1981, ApJ, 248, 291

Soszyñski, I., Udalski, A., Szymañski, M. K., et al. 2010, Acta Astronomica, 60, 165

Soszyński, I., Dziembowski, W. A., Udalski, A., et al. 2011, Acta Astronomica, 61, 1

Soszynski, I., Poleski, R., Udalski, A., et al. 2008, Acta Astronomica, 58, 163

Soszyński, I., Udalski, A., Szymański, M. K., et al. 2009, Acta Astronomica, 59, 1

Stellingwerf, R. F. 1978, ApJ, 224, 953

Stothers, R. B. 2006, ApJ, 652, 643

Subramaniam, A., \& Subramanian, S. 2009, A\&A, 503, L9

Szczygieł, D. M., \& Fabrycky, D. C. 2007, MNRAS, 377, 1263

Szczygieł, D. M., Pojmański, G., \& Pilecki, B. 2009, Acta Astronomica, 59, 137

Szeidl, B., \& Jurcsik, J. 2009, Communications in Asteroseismology, 160, 17

van Agt, S. L. T. J., \& Oosterhoff, P. T. 1959, Annalen van de Sterrewacht te Leiden, 21, 253

\section{Appendix A: DATA OF THE RESULT OF THE ANALYSIS.}


Table A.1 Parameters of light variation for the RR-SG stars. The full table is available in electronic form at the CDS.

\begin{tabular}{crrrrr}
\hline \hline ID & Period & $\Theta_{\mathrm{PDM}}$ & $A_{0}$ & $I$ & subtype \\
\hline 1 & 0.611821 & 0.1361 & 0.559 & 18.845 & RR0-SG \\
2 & 0.408541 & 0.1926 & 0.261 & 18.551 & RR1-SG \\
4 & 0.555120 & 0.0749 & 0.806 & 18.720 & RR0-SG \\
5 & 0.497251 & 0.0814 & 0.755 & 18.889 & RR0-SG \\
6 & 0.581477 & 0.1681 & 0.514 & 18.733 & RR0-SG \\
8 & 0.551528 & 0.0953 & 0.638 & 18.807 & RR0-SG \\
9 & 0.515681 & 0.1468 & 0.412 & 18.464 & RR0-SG \\
10 & 0.545564 & 0.1076 & 0.669 & 18.807 & RR0-SG \\
\hline
\end{tabular}

Table A.2 Parameters of light variation for the RR01 stars.

\begin{tabular}{crrrrrrrr}
\hline \hline ID & type & $P_{0}$ & $\Theta_{0}$ & $A_{0}$ & $I$ & $P_{1} / P_{0}$ & $\Theta_{1}$ & $A_{1} / A_{0}$ \\
\hline 3 & RR01 & 0.464248 & 0.61 & 0.169 & 18.816 & 0.7434 & 0.59 & 1.279 \\
53 & RR01 & 0.463814 & 0.55 & 0.158 & 18.768 & 0.7435 & 0.50 & 1.492 \\
157 & RR01 & 0.465272 & 0.57 & 0.169 & 18.890 & 0.7431 & 0.48 & 1.628 \\
174 & RR01 & 0.493743 & 0.57 & 0.178 & 18.782 & 0.7446 & 0.45 & 1.525 \\
249 & RR01 & 0.511593 & 0.62 & 0.121 & 18.664 & 0.7456 & 0.39 & 2.026 \\
264 & RR01 & 0.461786 & 0.51 & 0.235 & 18.886 & 0.7429 & 0.57 & 1.306 \\
330 & RR01 & 0.460572 & 0.47 & 0.247 & 18.850 & 0.7427 & 0.59 & 1.073 \\
332 & RR01 & 0.468276 & 0.73 & 0.106 & 18.845 & 0.7431 & 0.37 & 2.613 \\
382 & RR01 & 0.481604 & 0.84 & 0.128 & 18.692 & 0.7441 & 0.57 & 1.938 \\
483 & RR01 & 0.534026 & 0.65 & 0.098 & 18.482 & 0.7465 & 0.26 & 2.608 \\
528 & RR01 & 0.459523 & 0.60 & 0.234 & 18.611 & 0.7422 & 0.50 & 0.810 \\
531 & RR01 & 0.465609 & 0.65 & 0.146 & 18.669 & 0.7430 & 0.47 & 1.055 \\
558 & RR01 & 0.486225 & 0.52 & 0.164 & 18.773 & 0.7440 & 0.49 & 1.452 \\
622 & RR01 & 0.462159 & 0.47 & 0.213 & 18.874 & 0.7431 & 0.55 & 1.363 \\
642 & RR01 & 0.468788 & 0.57 & 0.295 & 18.824 & 0.7427 & 0.42 & 0.839 \\
\hline
\end{tabular}


Table A.3 Parameters of light variation for the RR-BL1 stars.

\begin{tabular}{|c|c|c|c|c|c|c|c|}
\hline ID & type & $f_{0}$ & $\Theta_{0}$ & $A_{0}$ & $I$ & $\Delta f$ & $A_{1} / A_{0}$ \\
\hline 7 & RR0 & 1.545554 & 0.50 & 0.106 & 18.056 & 0.010905 & 0.382 \\
\hline 20 & RR0 & 1.592310 & 0.15 & 0.747 & 18.597 & 0.020002 & 0.186 \\
\hline 78 & RR0 & 1.792749 & 0.27 & 0.515 & 18.975 & 0.018073 & 0.212 \\
\hline 103 & RR0 & 1.791820 & 0.42 & 0.419 & 18.770 & -0.001242 & 0.381 \\
\hline 125 & RR0 & 1.816095 & 0.37 & 0.423 & 18.809 & 0.009155 & 0.308 \\
\hline 191 & RR0 & 1.639542 & 0.27 & 0.443 & 18.598 & -0.000641 & 0.232 \\
\hline 193 & RR0 & 2.148450 & 0.25 & 0.563 & 18.751 & 0.005300 & 0.204 \\
\hline 196 & RR0 & 1.703644 & 0.17 & 0.388 & 18.496 & 0.001861 & 0.204 \\
\hline 217 & RR0 & 1.941551 & 0.33 & 0.477 & 18.910 & 0.025364 & 0.283 \\
\hline 230 & RR0 & 2.056198 & 0.24 & 0.594 & 18.768 & 0.022642 & 0.188 \\
\hline 239 & RR0 & 1.596092 & 0.45 & 0.265 & 18.696 & 0.017372 & 0.313 \\
\hline 253 & RR0 & 1.814577 & 0.13 & 0.583 & 18.586 & -0.004017 & 0.119 \\
\hline 261 & RR0 & 1.971605 & 0.24 & 0.530 & 18.886 & 0.007993 & 0.213 \\
\hline 275 & RR0 & 1.883136 & 0.17 & 0.485 & 18.627 & 0.035635 & 0.138 \\
\hline 280 & RR0 & 2.079711 & 0.23 & 0.664 & 19.010 & 0.043438 & 0.203 \\
\hline 283 & RR0 & 2.627460 & 0.31 & 0.491 & 19.285 & 0.016204 & 0.279 \\
\hline 311 & RR0 & 1.875675 & 0.16 & 0.645 & 18.733 & 0.030124 & 0.217 \\
\hline 313 & RR0 & 1.722907 & 0.37 & 0.393 & 18.765 & 0.010309 & 0.338 \\
\hline 325 & RR0 & 1.849624 & 0.27 & 0.543 & 18.882 & 0.010813 & 0.256 \\
\hline 347 & RR0 & 1.830031 & 0.12 & 0.641 & 18.692 & -0.003638 & 0.119 \\
\hline 377 & RR0 & 1.587357 & 0.31 & 0.293 & 18.736 & 0.030942 & 0.222 \\
\hline 427 & RR0 & 1.777755 & 0.25 & 0.502 & 18.721 & 0.022702 & 0.284 \\
\hline 444 & RR0 & 1.969073 & 0.18 & 0.667 & 18.862 & 0.002295 & 0.206 \\
\hline 464 & RR0 & 1.717309 & 0.28 & 0.377 & 18.766 & -0.009179 & 0.175 \\
\hline 469 & RR0 & 1.829113 & 0.25 & 0.592 & 18.883 & 0.016978 & 0.272 \\
\hline 517 & RR0 & 1.662463 & 0.42 & 0.227 & 18.537 & 0.026333 & 0.345 \\
\hline 579 & RR0 & 1.560733 & 0.21 & 0.493 & 18.602 & -0.023590 & 0.244 \\
\hline 597 & RR0 & 1.323251 & 0.37 & 0.197 & 18.507 & 0.011120 & 0.254 \\
\hline 624 & RR0 & 1.671056 & 0.27 & 0.360 & 18.391 & -0.007175 & 0.286 \\
\hline 639 & RR0 & 1.720961 & 0.25 & 0.354 & 18.651 & 0.019355 & 0.200 \\
\hline 645 & RR0 & 2.189412 & 0.15 & 0.619 & 18.762 & 0.014528 & 0.173 \\
\hline 652 & RR0 & 1.885687 & 0.24 & 0.422 & 18.703 & -0.009030 & 0.172 \\
\hline 95 & RR1 & 3.295831 & 0.37 & 0.352 & 19.148 & 0.000348 & 0.324 \\
\hline 107 & RR1 & 3.310811 & 0.64 & 0.110 & 18.716 & 0.072968 & 0.516 \\
\hline 126 & RR1 & 3.246747 & 0.78 & 0.087 & 18.737 & -0.072875 & 1.081 \\
\hline 164 & RR1 & 3.718042 & 0.66 & 0.154 & 19.057 & 0.132355 & 0.728 \\
\hline 198 & RR1 & 3.130009 & 0.47 & 0.198 & 18.747 & 0.000336 & 0.340 \\
\hline 317 & RR1 & 3.331974 & 0.47 & 0.206 & 18.784 & -0.128505 & 0.376 \\
\hline 322 & RR1 & 2.739613 & 0.63 & 0.178 & 18.734 & 0.001267 & 0.424 \\
\hline 542 & RR1 & 2.889081 & 0.75 & 0.073 & 18.700 & 0.156416 & 0.891 \\
\hline 613 & RR1 & 3.684208 & 0.63 & 0.142 & 19.009 & -0.072704 & 0.482 \\
\hline
\end{tabular}

Table A.4 Parameters of light variation for the RR-BL2 stars.

\begin{tabular}{ccccccccccc}
\hline \hline ID & type & $f_{0}$ & $\Theta_{0}$ & $A_{0}$ & $I$ & $\Delta f_{+}$ & $\Delta f_{-}$ & $A_{+} / A_{0}$ & $A_{-} / A_{0}$ & $A_{+} / A_{-}$ \\
\hline 165 & RR0 & 2.150372 & 0.27 & 0.567 & 18.753 & 0.022871 & 0.022862 & 0.290 & 0.182 & 1.593 \\
237 & RR0 & 2.044692 & 0.44 & 0.186 & 18.462 & 0.000405 & 0.000335 & 0.362 & 0.477 & 0.759 \\
441 & RR0 & 1.545543 & 0.32 & 0.283 & 18.699 & 0.016791 & 0.016839 & 0.260 & 0.214 & 1.215 \\
460 & RR0 & 1.594151 & 0.13 & 0.559 & 18.434 & 0.000376 & 0.000409 & 0.154 & 0.131 & 1.176 \\
594 & RR0 & 1.741086 & 0.42 & 0.367 & 18.641 & 0.000306 & 0.000422 & 0.373 & 0.233 & 1.601 \\
92 & RR1 & 2.988331 & 0.45 & 0.189 & 18.534 & 0.000337 & 0.000352 & 0.279 & 0.312 & 0.894 \\
227 & RR1 & 3.646038 & 0.38 & 0.265 & 19.032 & 0.000706 & 0.000708 & 0.269 & 0.272 & 0.989 \\
357 & RR1 & 3.560084 & 0.48 & 0.280 & 19.003 & 0.016841 & 0.016816 & 0.432 & 0.355 & 1.217 \\
529 & RR1 & 3.699046 & 0.69 & 0.158 & 18.977 & 0.082334 & 0.082303 & 0.571 & 0.574 & 0.995 \\
564 & RR1 & 3.031503 & 0.64 & 0.185 & 18.845 & 0.000433 & 0.000692 & 0.597 & 0.450 & 1.327 \\
580 & RR1 & 3.114803 & 0.41 & 0.224 & 18.773 & 0.006638 & 0.006642 & 0.292 & 0.256 & 1.141 \\
\hline
\end{tabular}


Table A.5 Parameters of light variation for the RR-MC stars.

\begin{tabular}{|c|c|c|c|c|c|c|c|c|c|c|c|c|}
\hline ID & type & $\Theta_{0}$ & $A_{0}$ & $I$ & $f_{0}$ & $\Delta f_{1}$ & $\Delta f_{2}$ & $\Delta f_{3}$ & $A_{1} / A_{0}$ & $A_{2} / A_{0}$ & $A_{3} / A_{0}$ & Notes \\
\hline 219 & RR0 & 0.50 & 0.319 & 18.675 & 1.903236 & -0.001661 & -0.000565 & 0.000533 & 0.568 & 0.341 & 0.303 & $\mathrm{a}$ \\
\hline 156 & RR1 & 0.53 & 0.224 & 18.810 & 2.645540 & 0.000353 & -0.001795 & -0.000964 & 0.428 & 0.335 & 0.330 & b \\
\hline 161 & RR1 & 0.52 & 0.222 & 18.742 & 2.836165 & -0.001872 & -0.000471 & -0.001482 & 0.558 & 0.452 & 0.498 & $\mathrm{c}$ \\
\hline 215 & RR1 & 0.75 & 0.176 & 18.933 & 2.817171 & 0.000403 & -0.000312 & -0.000804 & 0.772 & 0.468 & 0.480 & $\mathrm{~b}$ \\
\hline
\end{tabular}

Table A.6 Parameters of light variation for the RR-PC stars.

\begin{tabular}{|c|c|c|c|c|c|c|c|}
\hline ID & type & $f_{0}$ & $\overline{\Theta_{0}}$ & $A_{0}$ & $I$ & $\Delta f_{i}$ & $\overline{\overline{A_{i} / A_{0}}}$ \\
\hline 413 & RR0 & 1.717188 & 0.51 & 0.298 & 18.576 & -0.000309 & 0.590 \\
\hline 442 & RR0 & 1.791474 & 0.39 & 0.310 & 18.775 & 0.000204 & 0.278 \\
\hline 550 & RR0 & 1.864087 & 0.32 & 0.460 & 18.719 & -0.000301 & 0.272 \\
\hline 496 & RR1 & 3.656314 & 0.71 & 0.147 & 18.955 & -0.000279 & 0.503 \\
\hline 651 & RR1 & 3.309307 & 0.47 & 0.268 & 18.693 & 0.000244 & 0.378 \\
\hline \multirow[t]{2}{*}{247} & RR0 & 2.093590 & 0.39 & 0.483 & 18.999 & 0.000253 & 0.246 \\
\hline & & & & & & 0.000192 & 0.316 \\
\hline \multirow[t]{2}{*}{473} & RR1 & 2.447215 & 0.58 & 0.221 & 18.514 & 0.001115 & 0.459 \\
\hline & & & & & & 0.000148 & 0.319 \\
\hline \multirow[t]{2}{*}{581} & RR0 & 1.674840 & 0.38 & 0.388 & 18.730 & 0.000257 & 0.226 \\
\hline & & & & & & 0.000428 & 0.228 \\
\hline \multirow[t]{2}{*}{28} & RR1 & 2.981115 & 0.74 & 0.152 & 19.091 & -0.000213 & 0.686 \\
\hline & & & & & & -0.000516 & 0.648 \\
\hline \multirow[t]{2}{*}{67} & RR1 & 2.451110 & 0.45 & 0.253 & 18.554 & 0.000265 & 0.388 \\
\hline & & & & & & 0.000235 & 0.296 \\
\hline \multirow[t]{2}{*}{297} & RR1 & 3.127205 & 0.67 & 0.229 & 18.713 & 0.000274 & 0.516 \\
\hline & & & & & & 0.000127 & 0.501 \\
\hline \multirow[t]{2}{*}{586} & RR1 & 2.726628 & 0.47 & 0.210 & 18.815 & 0.000278 & 0.327 \\
\hline & & & & & & 0.000204 & 0.328 \\
\hline \multirow[t]{2}{*}{407} & RR0 & 2.141042 & 0.27 & 0.471 & 19.002 & 0.000609 & 0.276 \\
\hline & & & & & & 0.000192 & 0.219 \\
\hline \multirow[t]{3}{*}{312} & RR0 & 2.117445 & 0.56 & 0.384 & 18.902 & -0.000226 & 0.684 \\
\hline & & & & & & 0.000189 & 0.217 \\
\hline & & & & & & 0.004992 & 0.268 \\
\hline \multirow[t]{3}{*}{495} & RR0 & 2.075435 & 0.23 & 0.410 & 18.597 & 0.000258 & 0.173 \\
\hline & & & & & & -0.000092 & 0.172 \\
\hline & & & & & & 0.017395 & 0.161 \\
\hline \multirow[t]{3}{*}{501} & RR0 & 1.716627 & 0.42 & 0.355 & 18.582 & 0.000232 & 0.362 \\
\hline & & & & & & 0.020464 & 0.272 \\
\hline & & & & & & -0.000269 & 0.204 \\
\hline \multirow[t]{3}{*}{176} & RR1 & 2.743459 & 0.71 & 0.169 & 18.627 & -0.000926 & 0.459 \\
\hline & & & & & & -0.000240 & 0.436 \\
\hline & & & & & & -0.000642 & 0.492 \\
\hline \multirow[t]{3}{*}{519} & RR1 & 2.662524 & 0.40 & 0.249 & 18.678 & -0.000535 & 0.311 \\
\hline & & & & & & -0.000840 & 0.262 \\
\hline & & & & & & 0.000234 & 0.236 \\
\hline \multirow[t]{4}{*}{435} & RR0 & 1.639894 & 0.61 & 0.283 & 18.684 & 0.000207 & 0.513 \\
\hline & & & & & & 0.000161 & 0.633 \\
\hline & & & & & & 0.000090 & 0.280 \\
\hline & & & & & & 0.000354 & 0.336 \\
\hline \multirow[t]{4}{*}{596} & RR0 & 1.369461 & 0.56 & 0.279 & 18.713 & 0.000316 & 0.637 \\
\hline & & & & & & 0.000064 & 0.523 \\
\hline & & & & & & 0.000165 & 0.404 \\
\hline & & & & & & 0.000180 & 0.272 \\
\hline
\end{tabular}

